\title{
The Theories of Relativity and Bergson's Philosophy of Duration and Simultaneity During and After Einstein's 1922 Visit to Paris
}

\author{
C. S. Unnikrishnan \\ Tata Institute of Fundamental Research, Homi Bhabha Road, Mumbai 400005, India \\ E-mail address: unni@tifr.res.in
}

\begin{abstract}
In 1922, Albert Einstein visited Paris and interacted extensively with an illustrious section of the French academia. In overfilled sessions at the Collège de France and the Sorbonne, Einstein explained his theories of relativity, and prominent physicists, mathematicians and philosophers listened, debated, questioned and explored facets of relativity. The 1922 visit had its echoes in the life and works of many who participated, particularly decisive for Einstein and the philosopher Henri Bergson. This essay examines that eventful visit, focusing on the physical and logical aspects of Bergson's critique, with physics commentaries, linking prominent French physicists and mathematicians Langevin, Painlevé, Hadamard, Becquerel, Sagnac, and Kastler. I give particular attention to the logical and empirical accuracy of the physics issues involved, delineating Bergson's exact reasoning for his philosophical enthusiasm in Einstein's theory and for the ensuing critique. Bergson's philosophical stand on duration and simultaneity is reassessed in the context of later developments in cosmological physics as well as the wealth of empirical data involving comparison of atomic clocks. Finally we are led naturally to a surprising completion of the philosopher's program on universal time, duration and simultaneity, in harmony with the time of the physicist. In the appendices after the main text I also give the physics background and easily verifiable proofs for the assertions made in the text, pertaining to relativity, simultaneity and time dilation, clearly distinguishing beliefs and facts.

Keywords: Einstein's relativity, Bergson's philosophy, Time, Simultaneity, Twin paradox, Universe, Cosmic gravity, Cosmic Relativity,
\end{abstract}




\section{Introduction}

Einstein was invited for an academic visit to Paris in 1922 at the initiative of Paul Langevin, professor of experimental physics at the prestigious Collège de France. Einstein accepted the invitation, after an initial refusal for reasons of 'solidarity to his German colleagues', in an atmosphere of lingering nationalism after the First World War. He visited alone for a few days during March-April 1922. In several eagerly and enthusiastically attended sessions, French academia, from established professors to students, and a few from the general public got the opportunity to listen to Einstein's exposition of his theories of relativity. Then they actively sought clarifications and debated the physical, mathematical and philosophical aspects of the theories. On the whole, the well-publicised sessions at the Collège de France and the Sorbonne were very participatory and by and large friendly.

After the solar eclipse expeditions in 1919 that confirmed the gravitational bending of light, Einstein was a public figure all over the world. However, the wounds of the First World War had polarized the world and Europe, most strongly. Nationalistic attitudes permeated opinions on scientific matters as well. Einstein was a Swiss-German physicist working in Berlin, for the rest of the world. Admiration and opposition to Einstein's theories in France were influenced by these factors. The visit nevertheless happened by the conscious efforts of some on both sides, who insisted on reconciliation.

Perhaps the only incident that went beyond academic controversy was the planned boycott of the 'German' Einstein's scheduled visit to the Académie des Sciences by some members. Forewarned, Einstein cancelled the visit.

There are several descriptions of the 1922 visit and its analysis from different points of view, but most are centred on one of the sessions held at the Sorbonne on 6th April, 1922, arranged by the French Philosophical Society $[1,2,3,4,5]$. This visit was particularly engaging for historians because of the encounter between Albert Einstein and Henri Bergson. In the session at the Sorbonne, Bergson, the prominent and one of the influential philosophers of modern times and the professor of philosophy at the Collège de France, made a few critical and relevant comments on the notions of time and simultaneity in Einstein's Special (Restricted) Theory of Relativity. Bergson made these brief comments at the request of his colleagues, unplanned, despite his deep involvement and studies of the notions of time, duration and simultaneity [1]. His detailed opinions and stand on theses notions in the context of relativity and philosophy are explained with clarity 
in the monograph 'Durée et Simultanéité: à propos de la théorie d'Einstein' (abbreviated D-et-S, here), published later, in the same year [6]. At the session, he mentioned only the need to reconcile the common sense notions of universal time and simultaneity with Einstein's, in Special Relativity, and said he believed that this would be possible with a reinterpretation of the theory. Perhaps he was misunderstood, due to the brevity of the comments. The general impression was that he raised doubts on the physical relevance of Einstein's theory, and criticised it as a mere metaphysical thesis with mathematically defined, but unphysical, multitude of times. Anyway, it is a fact that Bergson's brief comments reverberated far outside the lecture hall of the Sorbonne, influencing even the Nobel committee's assessment of the theory; the 1921 prize was given to Einstein in December 1922, primarily for his theory of the photoelectric effect and there was explicit mention of the 'epistemological nature of the theory of relativity' in the presentation speech by the Nobel laureate scientist Svante Arrhenius: "Most discussion centres on his theory of relativity. This pertains essentially to epistemology and has therefore been the subject of lively debate in philosophical circles. It will be no secret that the famous philosopher Bergson in Paris has challenged this theory, while other philosophers have acclaimed it wholeheartedly."

In the next several sections, we retrace some instances of the 1922 visit in the context of the Einstein-Bergson interaction. Our interest here is not the general historical or socio-political aspects, which are well documented and analysed [1, 2, 3, 4]. Our focus is the physical theory, and its consistency and logical integrity, all in the backdrop of empirical facts. Thus, we necessarily include the events from the other sessions during the visit, at the Collège de France; there were four detailed sessions there that discussed Einstein's theories (March 31, April 3, 5, and 7, 1922). This then allows the proper understanding of Bergson's critique and its relation to his philosophy of time. The 1922 visit serves as an inclusive gathering that brought up and discussed the core ideas and views on the theories of relativity and the physics of time. Einstein and Bergson had continued interactions in the context of a transforming Europe and the world, especially in the context of the League of Nations. Their relationship was bound to be complex, both because of their individual views on the political issues involved, and due to their divergent views on time and simultaneity, which polarized their acquaintances as well. Due to the opinionated and polarized atmosphere, a careful and rigorous analysis of the physical and logical issues that were central to the debate during the Paris visit is lacking. Perhaps it was taken for 
granted by most commentators that Bergson's views were flawed because he failed to understand the relativity theory, new concepts of space and time, and their mathematical underpinning in the Lorentz transformations. Those who have read Bergson's work in Durée et Simultanéité (D-et-S) carefully, especially the sections on the Lorentz transformations and the discussion of synchronization of clocks and simultaneity, will realize that the factual situation is the opposite. His rigour as a mathematically competent philosopher addressing questions in a physical theory was stringent, and he was meticulous in his analysis. The 1922 encounter between Einstein and Bergson and the continued debates between Einstein's supporters and Bergson are documented in the edited volume 'Bergson and the Evolution of Physics' by P. A. Y. Gunter [7]. However, a careful analysis of the logical integrity of the positions of physics taken by the adversaries is still lacking.

We will not micro-analyse the actual exchanges during the 1922 sessions, because they were too brief and incomplete for a proper assessment of the positions and their reasons. Of course, these exchanges form the basis of the topics we discuss. We want to focus on the very issues that prompted Bergson to interfere - time, duration and simultaneity - in the context of important developments in the trajectory of physics. We limit our attention to a few of those who were present in these sessions, with occasional and necessary mention to Henri Poincaré, who was no more, except as a strong ethereal presence in the intellectual atmosphere discussing relativity and time. ${ }^{1}$ The characters, apart from Einstein and Bergson, are Paul Langevin (host and the master of ceremonies, and 'initiator' of the time dilation problem known later as the 'twin paradox', in 1911), Charles Nordmann (astronomer, physicist, author, and 'guide' to Einstein during the 1922 visit), Paul Painlevé (mathematician and politician who served as French war minister and prime minister), Jacques Hadamard (mathematician), Jean Becquerel (physicist and strong defender of Einstein's theory), Georges Sagnac (inventor of the Sagnac interferometer to prove in 1913 that 'ether existed' and Einstein's theory was flawed), and Alfred Kastler (then a first year student and later Nobel laureate for his work in optical pumping in atomic physics). We will

\footnotetext{
${ }^{1}$ Poincaré passed away in 1912 . He was the professor of mathematics, physics and astronomy at the Sorbonne, member of the French Academy of Sciences and the Académie Française, apart from serving as the Chief Engineer at the Corps des Mines. As a member of the Bureau de Longitudes he studied the problem of the global synchronization of time and made decisive founding contributions to the principle and theory of relativity.
} 
leave out many details and other characters, but we note that the EinsteinBergson affair echoed for several years through many people interested in physics, philosophy and politics, and its discussion even after a century may provoke unexpectedly strong and polarized reactions!

\section{Einstein, Bergson, and Time}

Bergson's studies on time, space, duration and simultaneity in the context of Einstein's theories were mature when the critical encounter happened. For Bergson, who had developed a philosophical framework for time and duration, such an analysis was on his natural philosophical itinerary. He stated about the origin of the work in the preface to the book,

We started it solely for our own benefit. We wanted to find out to what extent our concept of duration was compatible with Einstein's views on time.

Then he directly came to the important point that would define the focus,

Our concept of duration was really the translation of a direct and immediate experience. Without involving the hypothesis of a universal time as a necessary consequence, it harmonized quite naturally with this belief. It was therefore very nearly this popular idea with which we were going to confront Einstein's theory. And the way this theory appears to come into conflict with common opinion then rose to the fore...

In this section we discuss the physics background to the main points of contention between Einstein and Bergson. The new notions of time and simultaneity that evolved from H. A. Lorentz's theory of electrodynamic relativity formulated during 1895-1905 [8, 9], and from Einstein's own version of relativity in 1905 with differing interpretation [10], are at the basis of the debates. Lorentz's relativity theory, completed and interpreted by Poincaré, deals with the modification of the spatial and temporal intervals due to the motion through the 'ether', the hypothetical all-pervading medium for electromagnetic phenomena and the propagation of light waves. Einstein's theory also is based on identical mathematical structure, but with radically different interpretation, without the ether or any universal reference for motion. We explain the main elements to set the stage. There were two distinct 
issues that form the main body of the debate. One was the speed-dependent slowing down of clocks in motion, or the time dilation and resulting multitude of times in relativity theories. The other was the criterion for the temporal simultaneity of two events that happen at two spatial locations.

\subsection{Two theories of relativity}

We need to discuss briefly the main tenets of the Special Theory of Relativity (STR) and its consequences to duration and simultaneity before going ahead. STR originated in the fertile soil of experimental results in optics and electrodynamics, and the Lorentz-Poincaré Theory of Relativity (LPTR), developed during $1895-1905[8,9]$.

The definite completion of the theory of electrodynamics and the identification of light as the propagating waves of the electromagnetic fields were the high points in 19th century physics. It was assumed that the propagation of light required a universal medium, called the ether. The Galilean principle of relativity, that the state of uniform motion cannot be detected and distinguished from a state of rest, was at the basis of dynamics. This is related to the undetectability of pure 'space'. The question naturally arose whether the hypothetical ether could be detected by measuring the relative velocity of light while the laboratory on the earth was moving through the ether, just as one could detect the presence of the medium of air by measuring the velocity of sound relative to an observer moving and chasing the sound waves. However, the enormous velocity of light makes this task very difficult at multiple levels. The solution employing an interferometer, in which changes in distances much smaller than a millionth of a meter could be measured using waves of light, was invented by A. A. Michelson. From a refined experiment in 1887, Michelson and collaborator E. W. Morley announced the failure to detect the motion of the earth through the ether, in spite of sufficient sensitivity of the experiment [11]. It was as if the ether was as undetectable as the empty space; the Galilean principle of relativity seemed to be valid more generally, including all phenomena in mechanics and electrodynamics.

This null result implied an invariance property of Maxwell's equations of electrodynamics, similar to the invariance or the equivalence of Newton's laws in mechanics for all uniformly (inertially) moving observers. Starting with the ad hoc hypothesis of length contraction (that all extensions contract in the direction of motion by a certain fraction determined by the velocity through the ether), H. A. Lorentz eventually arrived at the 'Lorentz transformations' of spatial coordinates and time that achieved this invariance, while 
preserving the invisible ether and the Galilean notion of the variable relative speed of light [8]. Thus, in Lorentz's theory, the ether was like a universal absolute reference medium that remained undetectable. Real motion was motion relative to the ether. A measuring rod (scale) that moved relative to the ether contracted (length contraction) and the time measured by a moving clock progressed slower (time dilation). Henri Poincaré provided decisive mathematical completion to this theory and also clarified the meaning of the particular term of 'local time' in the Lorentz transformation, being linked to the synchronization of clocks at different places in the direction of the motion $[9,12]$. Poincaré stated the universal principle of relativity, applicable to all phenomena including gravitation.

That was when Einstein published his ideas of relativity in 1905, with the same principle of relativity as one of the postulates and a very different interpretation of the Lorentz transformations [10]. The principle of relativity is a generalization of Galilean relativity that asserts the impossibility to distinguish between the state of rest and the state of uniform motion. Einstein's generalization was to include all physical phenomena in its scope, just as Poincaré did. In the words of Max Planck [13],

The gist of this principle is: it is in no wise possible to detect the motion of a body relative to empty space; in fact, there is absolutely no physical sense in speaking of such a motion. If, therefore, two observers move with uniform but different velocities, then each of the two with exactly the same right may assert that with respect to empty space he is at rest, and there are no physical methods of measurement enabling us to decide in favour of the one or the other.

The different interpretation of the Lorentz transformations came from the second and the characteristic postulate of Einstein's theory - that the relative velocity of light is always an invariant constant, relative to any observer, moving or at rest. Once this non-intuitive feature is assumed, Lorentz transformations follow as a consequence. Of course, the null result of the Michelson-Morley experiment is then easily explained and all other consequences that Lorentz was struggling to understand as physically linked to the properties of the ether follow as the mathematical consequence of the theory without the ether. But there was a special price to pay; without any universal reference, the theory became symmetrical and reciprocal between 
any two observers in uniform relative motion. Any observer 'A' can claim being in a state of rest and that the other one ' $\mathrm{B}$ ' is moving, with the Lorentz modifications affecting the clocks and rulers of only the observer B. In turn, $\mathrm{B}$ can equally well claim that he is at rest and $\mathrm{A}$ is moving with exactly the same modifications happening only to A's clocks and rulers. The fastest clock and the longest ruler are always in the frame at rest. But, the frame at rest is equally $\mathrm{A}$ and $\mathrm{B}$, from their own frame. This is the point of departure in Einstein's theory regarding the notions of space and time postulated in physical theories until then. In other words, either A or B (or any other inertial frame) can claim the status of the special rest frame, which only the ether frame could claim earlier. This means that there is no meaning to the questions, 'which frame is really moving?', 'which clock and ruler are actually affected by motion?' etc. In the chosen rest frame, clocks and rulers are not affected. If this is seriously clashing with the intuitions and common sense built up over time, that is precisely the uneasiness that troubled many philosophers and even physicists.

So, in Einstein's theory, there is no physical reason for the modifications of time and length. In fact, there is no reason, in the sense of a causeeffect relation, because the cause - presumably motion - is not real, but only a relative notion in Einstein's theory. Relative inertial motion is a totally symmetrical notion among two reference frames, with equal right to claim the state of rest. What is demanded is only the consistency within each reference frame. Since observer A can only imagine what B measures, using theory, and cannot measure in B's place, mixing measurements does not happen. All they can do is to compare their physical measurements when they are at the same place - in infinitesimal proximity.

At the session in the Sorbonne, Bergson started his comments by expressing his admiration for Einstein's theory, which was 'new physics and in some respects, a new way of thinking'. He briefly stated his opinion that there was nothing incompatible between the common sense notion of a single universal time and Einstein's relativity [1];

Common sense believes in a unique time, the same for all beings and all things...the idea of a universal time, common to conscious beings and to things, is a simple hypothesis.

But it is a hypothesis that I believe to be founded and which, in my opinion, contains nothing incompatible with the theory of relativity. I cannot undertake to demonstrate this link. It would 
be necessary to study real duration and measurable time much more minutely than I have just done. It would be necessary to take the terms which enter the Lorentz' equations one by one and search for their concrete significance. Then one would find that the multiple times the theory of relativity deals with are far from all being able to pretend the same degree of reality...

But all that I cannot establish as regards time in general, I seek your permission to do, or at least glimpse, for the specific case of simultaneity. Here we will see easily that the relativistic point of view does not exclude the intuitive point of view, and even necessarily implies it.

Thus, only the issue of simultaneity was elaborated by Bergson (what he called a 'glimpse') in the discussion at the Sorbonne, due to paucity of time. We will thoroughly analyse the details in the section 'Simultaneity: Einstein vs Bergson'.

Even a cursory reading of D-et-S will convince anybody about the rigorous and clear nature of Bergson's writings. Penetrating the simple mathematical aspects of the relativity theories of Lorentz, Poincaré and Einstein was elementary for Bergson, because of his background in mathematics and proven ability and record, before he chose philosophy as his subject at the École Normale Supérieure. We have to keep in mind the important fact that in 1922, there was no direct experimental demonstration of time dilation as an observable physical effect. Hence, the dispute was not about whether effects like time dilation could happen in nature or not; the debate was strictly about whether the equivalent and symmetrical role to the two observers in relative motion characteristic of the Special Theory of Relativity was consistent with an actual asymmetrical physical effects deduced, favouring one of the clocks as slower than the other, without a justified reason. After all, it was known to Bergson (and discussed in detail in the first chapter of D-et-S) that the time dilation of clocks in motion is central to Lorentz's non-symmetrical theory of relativity in the ether, formulated well before Einstein's theory. If this crucial point is missed, then Bergson's stand is easily misunderstood and misrepresented.

\subsection{A mysterious postulate}

The propagation of light was central to the development of the theories of relativity. A feature that was of some uneasiness to many, if not most, 
was the fundamental characteristic postulate of Special Relativity, that the relative velocity of light was an invariant constant for all uniformly moving (inertial) observers. The discussions on simultaneity are intertwined with this topic. This may be best described, in the context of the visit, by quoting the uneasiness even in an admirer, friend, and supporter, Charles Nordmann [2],

However, ... there is still something infinitely troubling in the Einsteinian system. This system is admirably coherent, but it rests on a particular conception of the propagation of light. How are we to imagine that the propagation of a ray of light could be identical for an observer who flies away from it, and for an observer who rushes forward to meet it? If this is possible, it is in any case inconceivable to our customary mentality, and no matter how hard we try, we cannot make the mechanism and nature of that propagation intelligible.

It must be confessed that here lies a mystery which eludes us. The whole Einsteinian synthesis, as coherent as it is, rests on a mystery, exactly like the revealed religions.

The invariance of the relative speed of light, an 'infinitely troubling' mystery indeed, is the core postulate of Special Relativity. It is obvious from Nordmann's statement quoted above that the invariance of the velocity of light was not an empirically proven fact, contrary to the general erroneous (modern) belief that the 'null' result of the Michelson-Morley experiment of 1887 was the 'proof' of this 1905 postulate ${ }^{2}$ As in 1922 , even today we do not yet have any experimental confirmation of this 'postulate of revelation' (see later for details). However, this was not an issue of discussion or contention during the 1922 visit. Bergson understood clearly that the velocity of light was not a true invariant in Lorentz's ether relativity ('half-relativity', in his book), whereas it appeared to be a universal invariant in Einstein's theory, and accepted as a possibility, in spite of its anti-common sense nature. The difference in the nature of propagation of light in the two theories is at the very basis of their different notions of simultaneity of spatially separated

\footnotetext{
${ }^{2}$ The Michelson-Morley experiment was an optical proof for the principle of relativity. Its null result is obviously consistent with the Lorentz-Poincaré theory with the ether and Galilean light as well as with Einstein's theory. After all Lorentz explained the null result in 1894 using length contraction in ether.
} 
events. I stress again that it is an empirically unverified postulate, as evident in Nordmann's clear statement, contrary to the belief held by most physicists that it was verified in experiments even prior to Einstein's theory. Though it was not discussed during the 1922 sessions, it figured prominently in the published debate later, between Einstein's supporters and Bergson [7]. I quote from an article by André Metz (a 'faithful disciple' of Becquerel's ${ }^{3}$ ) in Revue de Philosophie (1924), "Einstein arrived at a different result by starting from an experimental fact...the isotropy of the propagation of light...the isotropy is firmly established, not only by the Michelson's experiments, but also, and especially, by all the experimental verifications of electromagnetic theory (Maxwell's theory)". Only people who have not read and understood the work of Fresnel, Fizeau, and Michelson, and the Lorentz-Poincaré relativity of 1895-1905, could make such blatantly fallacious statements. Consistency cannot be equated to empirical proof in science.

A. A. Michelson published a resume of his researches as a monograph 'Studies in Optics' in 1927 [14]. One chapter is dedicated to the measurements of the velocity of light, in which the Michelson-Morley experiment is not even mentioned! In that chapter, he clearly states that the constancy of the relative speed of light is a postulate of the theory of relativity. And in the chapter on 'Relativity', he discusses the Michelson-Morley experiment, correctly, as a test of the postulate of the general principle of relativity, which asserts the impossibility to detect the earth's motion through the ether. He concluded the chapter by saying that the result of his later experiment (with A. H. Gale) 'could be considered as additional evidence for relativity, or equally as evidence of a stationary ether'.

The hypothesis of the constancy of the speed of propagation of light relative to any inertial frame has remained untested experimentally to this day precisely because it is intertwined with the issue of synchronization and simultaneity of clocks in two different locations; the measurement of the velocity necessarily requires the measurement of duration that light takes to propagate from 'here' to 'there', and hence, between two clocks. Therefore, all measurements, direct and indirect, have been limited to the two-way speed in which light is brought back to the same point in space, to measure the duration of the two-way propagation, as in the Michelson-Morley experiment.

\footnotetext{
${ }^{3}$ Becquerel's role in the continued debate with Bergson on the twin paradox in Einstein's relativity is discussed later.
} 


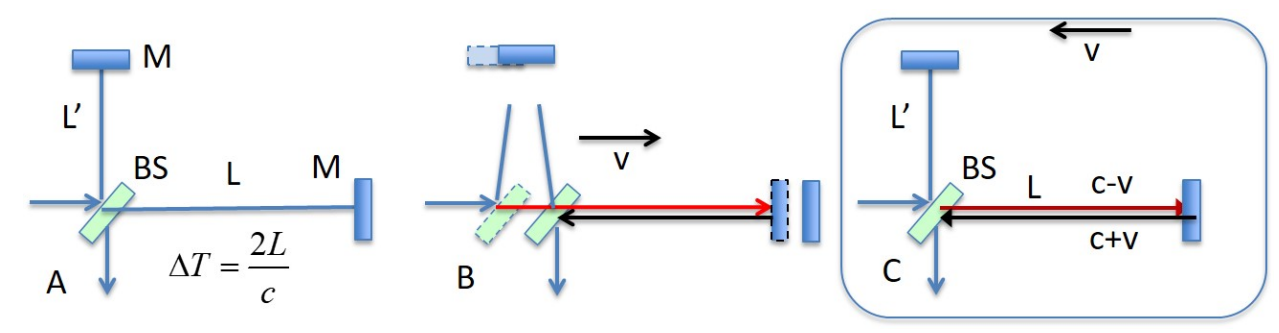

Figure 1: A: The Michelson Interferometer. Light waves travel from the beam splitter BS to the two mirrors $\mathrm{M}$ and back and exit overlapped, causing interference and 'fringes'. Any differential change in the distances to the mirrors results in a change of the intensity of the light output, or a shift of interference fringes. B: In the Michelson-Morley experiment, the interferometer is in motion with the earth's velocity $v$ while the light waves are on their two-way trip. As seen from a global frame, light traverses unequal up and down distances in the direction of the motion, as indicated with the red and black arrows. C: In the 'comoving frame' or the 'rest frame' of the earth and the interferometer, the relative velocity of light in the two directions is $c-v$ and $c+v$.

It is very easy to see why the Michelson-Morley experiment cannot decide on the isotropy of the propagation of light; the time taken in propagation over a length $L$ at speed $s$ is $L / s$. So, if the speed is not isotropic, but instead $c-v$ while light is in one direction and $c+v$ in the return path after reflection in the two-way experiment, total duration taken is

$$
T=\frac{L}{c-v}+\frac{L}{c+v}=\frac{2 L}{c\left(1-v^{2} / c^{2}\right)}
$$

Hence, the total duration is different from the isotropic value $2 L / c$ only by the small 'second order' term, $\left(1-v^{2} / c^{2}\right)$. If both length and time (clocks) are modified due to the motion (through ether, say) by factors $\sqrt{1-v^{2} / c^{2}}$, this excess factor of $\left(1-v^{2} / c^{2}\right)$ is exactly cancelled and we get $2 L / c$ as the duration even though the propagation of light is not isotropic! On the other hand if one postulates that the speed of light is isotropic in any frame, then there cannot be any modification of length or time in such frames. Either view is consistent with the Michelson-Morley experiment, making such experiments indecisive on this issue. It is an elementary fact, and there is no place for the erroneous belief.

It is obvious that a measurement of the one-way relative speed of light needs to tackle the hard problem of synchronizing two separated clocks. However, even the seasoned experts do not always realize the crucial point and one can see a number of well-cited publications claiming the verification of 
the postulate in measurements involving one-way propagation of light, with clocks at two different locations. The irony and embarrassment of the situation will be understood if it is noted that the need for such one-way measurements, to demarcate Einstein's theory from the Lorentz-Poincaré theory, was reiterated in a paper in the journal Physical Review Letters by M. Ruderfer in 1960 [15]. He suggested an experiment based on the newly discovered Mössbauer effect. Very soon Ruderfer published a vital erratum [16], when he understood the fundamental issue of the inseparability of the propagation delay and the duration measured with separated clocks. This fundamental 'catch' was already known and discussed by Poincaré, stressing the role of 'convention' in synchronization, but it is a subtle and deep issue that is easily missed, as history shows. Oblivious to the content of the erratum, researchers went ahead [17] and 'verified the fundamental postulate' and 'ruled out the Lorentz-Poincaré theory', while citing both Ruderfer's proposal paper as well as the nullifying erratum! And many are continuing in vain to refine these tests on light [18], still remaining in the dark about the interdependence of synchronization and time dilation of separated clocks in moving frames, and the inability of such tests to decide between the two kinds of theories, as pointed out clearly by Ruderfer.

\subsection{Bergson and Einstein's theory}

Simultaneity of physical events as a fundamental premise was discussed in detail by Einstein, with the scenario of an observer on a platform and another in a moving train, in his expository monograph on the theories of relativity (1916), 'Relativity: The Special and General Theory' [19]. Bergson meticulously analysed Einstein's conception of simultaneity, in D-et-S. Contrasting the common notion of simultaneity with that in the theory of relativity, the philosopher hoped that there would be convergence of the concepts. But, it seemed that there was irreconcilable discord between the two views. Bergson pointed out the inconsistency between the conclusions arrived at by Einstein and the symmetric reciprocity of Special Relativity (this easily verifiable point has been discussed by a few others ever since, but generally ignored. We will discuss our transparent proof later, in the section on simultaneity). He also discussed in great detail the problem of the two brothers and their clocks (the twin paradox), and the issue of multitude of times in relativity. The relativists defended strongly and vigorously ('more Einsteinian than Einstein'), concluding that Bergson misunderstood the theory and the Lorentz transformations. The relativists went further and 
concluded that the edifice of the Bergsonian philosophy of time and duration crumbled due to his mistaken views about real time, which was the time of physics as well as of Einstein's theory.

There is no need to examine in detail the philosopher's distinction between the notions of 'time' and 'duration' in our discussion. But it is important to make a clarifying remark. Only the 'duration of time' is physically (and psychologically) sensible. Duration is the measure of elapsed time, and all physical phenomena are events measuring some duration. Thus, the concepts of continuity and extension are naturally built into the notion of duration. As Bergson wrote in D-et-S, his notion of duration harmonized naturally with the hypothesis of a universal time. Bergson's examination of the physical theory was to see whether it was in harmony with the time in physics.

Bergson's position on Einstein's theory and the motivation for his hopeful admiration for the theory is clearly stated in D-et-S, without any scope for ambiguity, right after the first chapter 'Half Relativity' and two pages into the second, 'Complete Relativity'. In 'Half Relativity' he analyzed and interpreted the terms in the Lorentz transformations, one by one (as he indicated in the session at the Sorbonne), and discussed in detail the synchronization of clocks and its dependence on the protocol with the propagation of signals. This was in the context of the Lorentz's theory, and the interpretation was close to that of Poincaré, though Bergson did not (surprisingly, for me) mention Poincaré in D-et-S. With Lorentz's theory and its ether as the privileged frame, there was one system (of reference) $\mathrm{S}$ that possessed the master clock and time, and in a system S' that was relatively moving there was a different, dilated real time. However, there was no physical way to know whether one was moving or not through the insensible ether's privileged frame! Hence, Lorentz's relativity was at direct conflict with a single universal time central to Bergson's philosophy. With Einstein's relativity, all observers S, S', S"..., were deemed equivalent with equal right to claim the state of rest; there was multitude of times, now apparent and interchangeable, with the dilation always affecting the 'other' clocks. Therefore, Bergson hoped and reasoned that these mathematical multitude of times could not be identical to the real time, and the whole picture could be made consistent with the Bergsonian preference for a single universal real time. This is the crux of Bergson's evaluation and interpretation of Einstein's relativity.

As Bergson stated in the preface to D-et-S, "we started it solely for our own benefit". We see that Bergson approached the theory as a charmed 
suitor and not as a critique, but ended up criticizing the theory to bring out what he thought was its interpretational essence that would harmonize with a universal real time. Lorentz's theory, with its real time dilation for real motion in invisible ether, was in direct conflict with Bergson's concept of time, whereas Einstein's theory without an absolute reference for motion gave him the hope of reconciling the notions of time in physical relativity and time of the philosopher, and indeed of common intuition. With no empirical method known to choose one theory over the other, the philosopher had a choice in Einstein's theory, with the possibility of reconciliation with proper interpretation. If Lorentz-Poincaré theory were the only theory, Bergson's thesis on universal duration and simultaneity would already be invalidated, being in conflict with the only physical theory of empirical experience. This is the core point concerning Bergson's interest and engagement, as a philosopher, in the physical theories of relativistic time.

\section{Irrelevant Common Sense}

When we discuss the Special Theory of Relativity, we will need to disregard certain kinds of objections that make sense from the point of view of common sense, but that are not immediately useful in the analysis of the theory. Two instances can give a flavour of the situation.

I am an observer at rest in STR - I am always at rest in my frame in STR, by definition; that is my rest frame. I see several things moving past. Surprisingly, they all are moving in the same direction and with the same velocity. An analogous thing happened in history when it was noticed that the entire system of stars and the globes of the solar system alike were moving as an ensemble from east to west every day. This was finally simplified by realizing that the earth is 'really' rotating, while stars stayed more or less fixed. So, should I conclude from common sense that I am the one who is really moving and physical effects should happen only to my clocks and measuring rods, if at all? STR insists that one is not justified to draw such a conclusion - there is nothing called real uniform motion. Every inertial motion is equivalent to a state of rest. In one's rest frame, however humble, the rest of the universe is moving; the rest of the clocks and rulers change.

More down to earth, I see a flock of birds in the standard formation, but all going backwards. I know that it is impossible. But, the physical theory of space and time doesn't care. As physical objects, the birds are in motion, while I am at rest. To the question, 'who's clock is going slower?', the theory 
gives the answer: the internal clocks of the birds are running slower, whether they are flying forward or backward. If they appear still, despite the flutter, they are also at rest and there is no time dilation. In the ether-relativity, it would have been different; the appearance of the 'unnatural' motion of the birds must be due to my faster real motion relative to the ether. Can I say that the birds that are 'really' moving forward slower than me, and hence appearing moving backwards, must also be ageing faster relative to me, guided by common sense? Einstein's theory answers in the negative, because it is based on kinematics in the empty space. Since durations cannot be compared unless I mark the time on the clocks twice - at the start and at the end, at the same place, there seems no way to verify my common sense guess with birds that have flown past. (However, see appendix II).

So, the distinction is clear. In LPTR, modifications of lengths and durations are real physical effects that increase with the velocity relative to the ether. In STR, these modifications depend on the relative motion between the frames and the choice of being at rest is left free; only clocks and rulers external to our rest frame are affected. We are at rest, in our frame, and nothing happens to our clocks and rulers. Anybody's clock can be chosen to run the fastest by merely occupying his frame, and claiming the state of rest. There are as many fastest (or 'normal') clocks as there are observers hence, the reciprocal multitude of times of the Special Relativity. What is not noticed is a glaring logical gap; if one could claim a state of rest in situations of relative inertial motion, then one can also claim a state of motion at will, making the physics of clocks arbitrary. Given a value for the relative velocity between two frames, there is an infinity of possible partitions of individual velocities between the frames. But, that is the topic of discussion in a separate paper on the logical structure of the theories of relativity [20].

Clearly, the philosopher might not have been worried about the conflict with common sense in the way of the examples we mentioned. He was worried about the meaning of the assertions of the theory and whether they rigourously and logically follow from the symmetrical structure of the theory, with its temporal multitude. Einstein, on the other hand, was concerned only about the consistency of the theory with what was measurable. He was also aware that his theory gave the same predictions as the Lorentz-Poincaré ether-based theory in situations of common physical measurements. Above all, the confirmed predictions of the General Theory of Relativity (GTR) for the gravitational physics of the motion of the planets and the bending of light near the sun were an indication of the correctness of his approach, and 
was perhaps the basis of his confident modesty.

\section{Langevin's Twin Paradox}

The Special Theory of Relativity was projected as a theory of space and time, rather than merely as a theory of motional modifications of distances, durations, and related physical quantities, after H. Minkowski's mathematical formulation in which 'time' was depicted as a fourth dimension, along with the three spatial dimensions [21]. However, the fact that this dimension had the imaginary nature, in the mathematical sense, confined the geometrical theory within the circle of physicists and mathematicians (in older texts, it was depicted as $(i c) t$, where $i=\sqrt{-1}$ ). It was through a lecture by Langevin at the International Congress of Philosophy in Bologna in 1911 [22] that many philosophers were jolted into a discussion about the multitude of times in the physical world, one each and one's own for every inertial observer. Bergson was a speaker at the congress in Bologna, where he spoke about 'Philosophical Intuition'. There, Langevin mentioned about time dilation of transported clocks in Einstein's theory, and discussed two concrete examples. One was about the comparison of the lifetimes of two samples of radioactive Radium in relative motion, and another was the scenario of the space traveller. He said,

Imagine a laboratory attached to the Earth, which motion can be considered as uniform translation, and in this laboratory there are two perfectly identical samples of radium. What we know about the spontaneous evolution of radioactive materials allows us to say that if these samples are kept in the laboratory, they will lose both their activity the same way over time and their activities remain continuously equal. But then send one of these samples with a sufficiently high velocity and then bring it back to the laboratory; this requires that at least at certain times this sample has undergone accelerations. We can say that on return, its proper time between departure and return is less than the measured time interval between these events by observers attached to the laboratory, so that it has less evolved than the other sample and therefore it will be more active than the latter; it will have aged less, having been more agitated.

As the next example of 'lived durations' of the traveller he said, 
For this it is sufficient that our traveller consents to be locked in a projectile that would be launched from Earth with a velocity sufficiently close to that of light but lower, which is physically possible, while arranging an encounter with, for example, a star that happens after one year of the traveller's life, and which sends him back to Earth with the same velocity. Returned to Earth he has aged two years, then he leaves his ark and finds our world two hundred years older, if his velocity remained in the range of only one twenty-thousandth less than the velocity of light. The most established experimental facts of physics allow us to assert that this would actually be so.

This second example evolved into the fable of the 'twin paradox' of relativity. One of the twin brothers go out in a long and fast space-voyage. When he returns home, he has aged much less than his brother who stayed back. What is the explanation for the unequal ageing? Langevin was aware and careful about the fact that, in Special Relativity, any observer can claim the state of rest and the special relativistic ageing was symmetric, unless some kinematical asymmetry was invoked. He cited the asymmetry of the accelerations of one of them, required to make the return trip for the eventual comparison at the same place. He stated that the one who had aged the least is the one who had suffered the greatest accelerations. He devised a scheme of light signals for the continuous communication between the traveller and the earth station and demonstrated the asymmetry as equivalent to the asymmetry of Doppler shifts of the light signals.

However, there was nothing in the inertial physical basis or the mathematical structure of Special Relativity that could justify the assertion, 'one who had aged the least is the one who had suffered the greatest accelerations'. Those who do not agree with Langevin's unjustified reasoning would naturally interpret this situation as a paradox - hence the twin paradox, where one person remains on the earth (A) and his twin brother (B) is the space traveller. One would argue that in the space traveller's rest frame, it is the earth dweller who is relatively moving and Einstein's theory (through the Lorentz transformation on duration) predicts that the earth clocks (A) run slower, irrespective of the direction of travel and without regard to whether they are continuously monitored by B. There is nothing in Special Relativity which demands a special treatment while turning back - that is a small duration compared to years of travel. So, relative to B, it is $\mathrm{A}$ who ages 

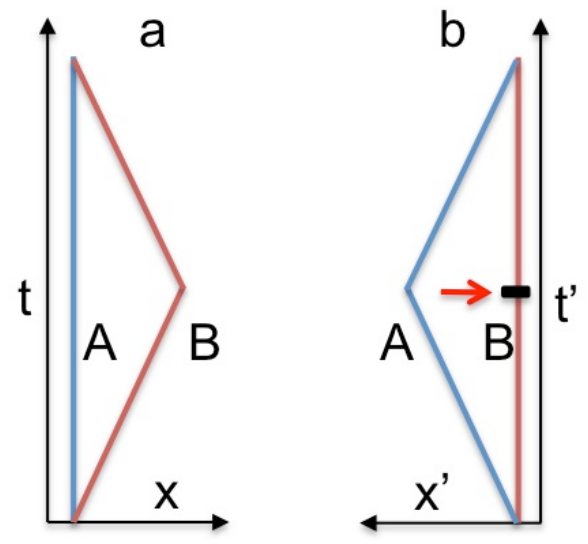

Figure 2: Space-Time diagrams of the twin clock problem. a) The diagram from the rest frame of A, with vertical 'world line' in blue. B moves out in space and returns. The 'length' of B's world line and the duration measured in his clock are shorter, due to the negative contribution from the motion in space. b) Relativity from B's rest frame. Now B has the vertical world line and A moves out in space and returns. B feels a squeeze around the midpoint, indistinguishable from gravity, for a short duration (red arrow). A's world line is shorter and the proper duration in his clock, is less. The situation is entirely symmetrical, save for the brief period of gravity in B's world frame. There are many who draw only the diagram (a) and conclude that 'obviously B ages less', without any mention of the diagram (b), acceleration, or gravity.

slower, whereas relative to A, it is B who ages slower. Since both have equal special relativistic right to claim the state of rest, both are right and the only logical conclusion, without invoking any new theory, is that neither will be slower or faster. Hence, time dilation in Special Relativity must be an unphysical illusion - that is the essence of the twin paradox and the critique of Special Relativity. This problem doesn't arise in Lorentz-Poincaré theory, where time dilations occur only while moving relative to the ether - the situation is asymmetric from the start to end, without accelerations. In any case, the Special Theory of Relativity does not specify a reason for a clock that underwent a brief period of noninertial motion to go slower than the one that didn't.

To be precise, the physical time (duration) recorded by a moving clock in Einstein's theory is given by a simple formula,

$$
(d \tau)^{2}=(d t)^{2}-(d x / c)^{2}
$$

where $d t$ is the conventional duration of the familiar Galilean time and $d x$ the 
distance moved. This equation defines the 'geometry' of STR. The difference from the familiar Pythagoras triangle formula is the negative sign. So, the 'time base' of the space-time triangle, corresponding to the duration in the rest frame, is longer than the sum of the other two sides (see Fig. 2). In the frame of the clock, it is at rest and $d x=0$; then the physical time and the clock time are identical, and flow fastest. For any moving clock, the physical time $d \tau$ is shorter than $d t$ because of the subtractive contribution from moving the distance $d x$. While the rest frame clock C1 marks 100 seconds, another clock C2 that has relatively moved by a 1000 kilometres marks about 55 nanoseconds less than $100 \mathrm{~s}$, as theoretically calculated from the $C 1$ rest frame. In contrast, $\mathrm{C} 2$ is at rest in its frame, and while it marks $100 \mathrm{~s}, \mathrm{C} 2$ has moved $1000 \mathrm{~km}$ and records 55 nanoseconds short of $100 \mathrm{~s}$, as calculated from C2 frame. Since any of the inertial observers has the right to claim the state of rest in the theory, $d x=0$, after rejecting a deciding privileged frame like the ether, the clocks in that frame run the fastest, and all other clocks slower. The arbitrary choice of the 'immobile' frame with complete equal rights is the source of the controversy. Lorentz and Poincaré didn't have to face this difficulty because there was the special frame of the ether in which the immobile clocks had the fastest rate, and any clock moving in the ether was slower; there was no reciprocity.

\subsection{Divergent views: \\ Langevin, Einstein, Painlevé, and Becquerel}

Langevin's 1911 solution remains as one of the popular answers to the paradox in modern text books. ${ }^{4}$ Yet, most of the discussions do not spell out how exactly the asymmetry of acceleration translates to an observable physical effect. Instead, nearly everybody insists that the paradox is cleared entirely within the Special Theory of Relativity. Few realize that Einstein had rejected such a resolution as incorrect in 1918, four years before the Paris visit and long after the assertion on asymmetric time dilation was made in the 1905 paper on the Special Theory of Relativity [10]. He admitted in the paper published in Die Naturwissenschaften [23] that the relativists were 'evading the issue' and the solution required going beyond the special theory,

\footnotetext{
${ }^{4}$ Different text books on Special Relativity offer three or four physically different solutions to the twin paradox, signalling the perennial confusion and the lack of consensus. These different reasons are Doppler effect, Change of simultaneity, Acceleration asymmmetry, and Gravitational time dilation in accelerated frames.
} 
because the situation was symmetric in the theory! Most textbook authors who have written on time dilation in relativity are not even aware of this paper. Einstein's much awaited and delayed resolution of the paradox was that the pseudo-gravitational field experienced during the acceleration phase (during the reversal of the velocity for the return journey) resulted in sufficient amount of gravitational modification of relative time in the spaceship frame according to his new theory of gravity - the General Theory of Relativity - that there was really no contradiction or paradox [23]. This was indeed his first definitive and quantitative response to the twin paradox. Both Nordmann and Kastler mention the need for General Relativity input to solve the paradox, in their memoirs of the 1922 visit. In contrast, most text books and monographs do not even mention Einstein's asserted solution and pretend that the correct solution is entirely within Special Relativity. We sketch Einstein's simple gravitational solution to the twin paradox in the appendix I.

The paradox was vigorously discussed during the 1922 visit, brought to debate by the insistent Painlevé in one session at the Collège de France [2, 34]. The summary from Nordmann's record is that Einstein's replies to the queries were satisfactory to the gathering and Painlevé. But, Einstein seemed to have chosen to keep quiet on the necessity of invoking General Relativity and the gravitational time dilation to justify the asymmetric time dilation, and to resolve the paradox that arose in the special theory. We will see that while this did not affect Einstein's reputation, his physicist defenders like Jean Becquerel continued to use biased and incorrect arguments entirely within Special Relativity to justify the asymmetric dilation. The irony is exemplified by a letter that Becquerel sent to Bergson, in which Becquerel 'explained away' the asymmetric time dilation in Special Relativity without discussing acceleration, even during the reversal of the journey. Little did Becquerel realize that his discussion of time dilation would become a record of how even distinguished physicists misunderstood the Special Theory of Relativity and its criticisms, as Bergson spotted and exposed in an appendix to D-et-S, second edition.

Becquerel's letter was reproduced (to represent his views faithfully) in the later editions of Duration and Simultaneity, in the first appendix [24]. For Bergson, it was easy to spot and show that Becquerel was inadvertently using a theory of relativity with a preferred frame and not Special Relativity in his 'demonstration' (Bergson did not name the 'most distinguished physicist' in his book). 


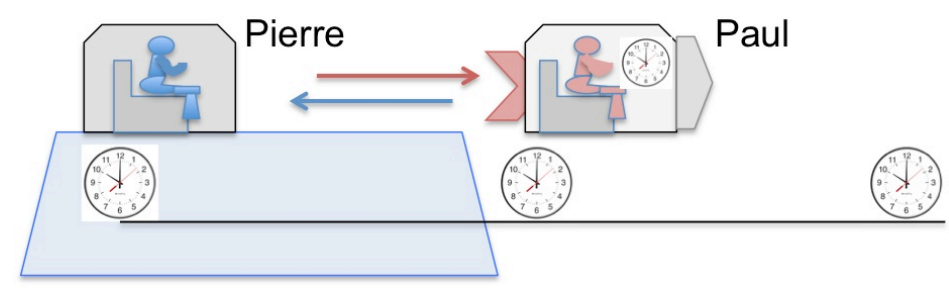

Figure 3: Becquerel's version of the scenario of the twins, where the asymmetrical ageing is deduced even for the inertial phases, is indicated. Clocks are synchronized to Pierre's clock. Becquerel asserted that Paul would see the clocks that move past him read more time relative to his clock, at rest with him, and hence Pierre would age more.

Briefly, Becquerel discussed the standard twin problem, with one of them on earth and the other blasting off at high speed. In those French discussions, they are named Pierre and Paul. In Becquerel's scheme without accelerations, there are clocks placed throughout the trajectory, all synchronized with the earth clock. Paul adjusts his clock to read the same time as Pierre's as the two meet in their paths. What is then simpler than Paul's just comparing his clock with the nearest 'earth synchronized clock (ESC)' on his path to find out who ages slower? Becquerel concludes from the Lorentz transformations and Special Relativity that Paul's clock always reads less than ESC. So, according to Becquerel, Paul in his rest frame would conclude that he was ageing slower after he separates from Pierre, even without any asymmetry introduced. Since the total time should be twice that for half the journey, once it is argued that Paul remained younger than Pierre during half the journey, Becquerel concluded that Paul would return younger. Becquerel analyzed the entire journey from the frame of Paul in the spacecraft, i.e., Paul's rest frame. And, surprisingly for a physicist, he concluded that Paul would see less duration elapsed in his clock, compared to Pierre's, even during the inertial journey. So, Becquerel, in effect, assumes that Paul is the 'really moving guy', relative to Pierre as well as in Paul's own reckoning. He forgets the most important aspect of Special Relativity that the clock in its own rest frame goes the fastest and the (proper) duration recorded in any relatively moving clock is less. Therefore, irrespective of what the other passing clocks read, Pierre must have clocked less time, having moved some relative distance. Remember the formula for the elemental relativistic durations, $(d \tau)^{2}=(d t)^{2}-(d x / c)^{2}$. It was an elementary mistake from Becquerel. I think his confusion was because he mistook the clock's local time readings 
at the spatial locations of comparisons (as given by Lorentz transformation formula) as identical to Pierre's elapsed duration.

In contrast, Bergson's analysis is clear; concerning the outward and inward journeys he says,

But, from the standpoint of theory of relativity, there is no longer any absolute motion or absolute immobility. The first of the two phases just mentioned then becomes an increasing distance apart between Pierre and Paul; and the second a decreasing one...The symmetry is perfect...their situations are interchangeable.

Then Bergson shows that, using the same Lorentz transformations, it is Pierre's clock that runs slower, as observed from the rest frame of Paul's, during the inertial portions of the journey. This was exactly the same conclusion Einstein emphasized in his 1918 twin paradox paper, for the inertial portion of the journey. In summary, Becquerel made the grave mistake to argue that the clocks in the rest frame $\mathrm{S}$ were slower than the clocks in a relatively moving system $S^{\prime}$ in Special Relativity. This error was carried by some other French supporters of Einstein's, like André Metz, in their criticisms of Bergson, but we will not go into those details. ${ }^{5}$ Becquerel's error is there in the reprinted letter in D-et-S for all to see and easily verify. Ironically, those who claim that the twin paradox is resolved entirely within Special Relativity are inadvertently asserting that Einstein's solution in 1918 [23] and the Equivalence Principle (which he used in the solution) are incorrect, without citing any reason.

\subsection{The need for logical rigour and consistency}

Discussions on the twin paradox often slip philosophically and logically in distinguishing time dilation in nature and time dilation in a particular theory. Whether a theory represents the phenomenon (of asymmetric time dilation here) in its logico-mathematical structure depends on the nature of the theory, irrespective of whether there is such a phenomenon in nature. Bergson, being a philosopher, was arguing that the symmetric and reciprocal theory of Special Relativity could not have asymmetrical multitude of real times. The same point was made by Einstein himself in his 1918 paper on

\footnotetext{
${ }^{5}$ P. A. Y. Gunter's edited volume contains translations of articles by Metz and Bergson's replies, in Revue de Philosophie (1924).
} 
the twin paradox. At a time when direct experimental evidence for the time dilation was not available, further questions on the reality and the physical reason for the phenomenon were empirically undecidable. Note that nobody really debated about the gravitational time dilation as a paradox in days when it was not verified, though the effect was known for as long as the twin paradox of STR itself; there was no reciprocity about two different heights in a gravitational field. But, the problem special relativists faced was that if they admitted that the time dilation was symmetric in STR, the theory would become vacuous.

Finally when direct experimental evidence for time dilation became available in 1941, first in the dilation of the life time of cosmic ray muons [25, 26], it was clear that acceleration had no direct role to play in time dilation; the energy loss of these charged particles subject them to enormous deceleration (more than a trillion times the gravity on earth), but the time dilation measured is simply related to the velocity only. However, acceleration that happens only after half the journey is invoked as the agent of asymmetrical time dilation in all modern discussions of the twin paradox. Finally, even a layman may be able to ask a 'most distinguished physicist' how would acceleration play any role at all if one decides to freeze the clock during the brief durations of acceleration and restart it where it was stopped after the acceleration! After all, the distant clock with Pierre cannot get affected by the accelerations of Paul [27].

Bergson's view of the twin clock problem within Special Relativity was as we stated already; in the completely symmetric theory, there was no room for the alleged asymmetry. The analogy he used was the appearance of one of the twins to the other when they are nearby and when they are far. At a distance, the person will appear as a midget, but who of the two is appearing as the midget will depend on who's view is taken as privileged. Paul will appear as midget from Pierre's point of view and Pierre will be judged as midget from Paul's point of view. At the end, when both can be together the difference in size dissolves away. The apparent plurality of durations is similar and in Bergson's view, there is no real relative time dilation in Special Relativity. The corollary is that if time dilation was known to be a fact of nature, Bergson would have probably argued that the explanation was not in Special Relativity, but in a different theory (like Lorentz's theory) with the appropriate inherent asymmetry in inertial motion.

We conclude this section on time dilation with one ironical empirical outcome and a related incident of quenched criticism. An experiment on 
time dilation 'on a set of twins' was done with Caesium atomic clocks in 1972 [28]. (In this experiment, there were also gravitational effects on the rate of clocks, but the value was known from General Relativity and could be subtracted to get the ageing due to only the relative motion). One set of the twin clocks (Pierre) remained in the Naval Observatory in Washington DC, while the other set (Paul), who made a trip in an aeroplane via the West Coast, Guam, Bombay, Rome,... arrived back in Washington expecting an older Pierre, who remained in his room, as in the relativity fables. But, Paul found Pierre to be younger than him! What does it say for the theory? This experimental result should have been an eye-opener which revealed that the time dilation was not decided by relative velocity, but it wasn't to be. This unexpected turn made the experimenters to invent an imaginary frame-fairy watching all this from an encompassing space, much like a privileged frame. In effect, they used a limited version of Lorentz's ether relativity to explain the experimental results, but attributed the credit to Einstein's theory.

Everybody wasn't convinced. Louis Essen, credited as the inventor of the practical Caesium atomic clock at the National Physical Laboratory in England (1955), had been involved in many precision tests of the theories of relativity. He was the force behind the current definition of the standard second, in terms of the atomic transition frequency in Caesium. His method of deducing the velocity of light from the measurement of its frequency and wavelength in a resonator is the modern method for fixing the velocity of light. ${ }^{6}$ In the early seventies he turned a critique of the theory, arguing that it was just a transformation theory on units and not a physical theory. He was critical of reducing the two fundamental units of length and time into just one, with the speed of light 'c' as the conversion factor. His thoughts on relativity were expressed in the tiny monograph, 'The Special Theory of Relativity: a Critical Analysis' [29]. Soon, he was excluded from publishing his criticisms on these topics, especially on the transported Caesium atomic clocks experiments.

\footnotetext{
${ }^{6}$ The most precise value of the speed of light is not really a measured of the 'speed' from the propagation of light. It is the product of two precision measurements of the frequency and wavelength $(c=f \lambda)$ of a standing wave in a cavity resonator.
} 


\section{Simultaneity: Einstein vs. Bergson}

Bergson's brief comments at the Sorbonne during Einstein's visit were mostly focused on the notion of simultaneity, after a brief mention of multitude of time in the theory. The concept of simultaneity is of central importance in Special Relativity, being an essential prerequisite for defining synchronization of clocks and the measurement of time. According to Einstein, the common sense notion of simultaneity was defective and could not be maintained in a physical theory. Events happening in two spatial locations, appearing simultaneous to one observer because he perceive them at the same time on his clock, will not be in general simultaneous to another observer who is moving relative to the first. The time of an event can be reliably attributed only by consulting a clock adjacent to the event. Then, one has to specify a method to synchronize such clocks at different locations, to build a consistent physical theory. That was how the constancy of the velocity of light in all inertial frames became the pivotal point of relativity. If all clocks are synchronized in the rest frame of the clocks using light, they are all properly synchronized, independent of any common uniform velocity they may have. In other words, they are synchronized as if they are all at rest.

The common sense notion of simultaneity is linked to the notions of a universal time, and also the psychologically 'felt' time and duration. Bergson referred to a temporal 'cut' of the universe of events, invoking absolute simultaneity as a legitimate concept. He was objecting to its denial citing the obvious point that 'news' of two events from a distance, brought by the messenger light, as simultaneous to one observer was not simultaneous to another who intercepted the messenger signals at another location because of motion. Bergson was trying to reconcile the common sense notion and the role of the concept in the physical theory. Also, when one says that the event is labelled with a nearby clock, one will have to make precise the notion 'nearby'. He asserted that these considerations at a microscopic level need further clarification with more precision ('we are more Einsteinian than you, Mr. Einstein' [1]). As before, the main point of contention was not whether simultaneity for one could become succession for another observer in natural phenomena, but whether that happens in Einstein's theory with its peculiar postulate. That it happens in Lorentz's theory is discussed by Bergson himself (in D-et-S, chapter 1), without ambiguity. Does it happen in Einstein's Special Relativity? 
We know that the sound of two bells originating in two locations $\mathrm{A}$ and $\mathrm{B}$, equally distant to our left and right, will be heard simultaneously, while standing motionless at the midpoint. Another person co-located with us when the bells were sounded, but moving relative to us, will hear them one after the other, i.e., in succession, because he has moved away from the midpoint. The Galilean nature of the propagation of sound waves, related to the existence of the medium as a privileged frame, is the reason for this familiar fact.

During his intervention, Bergson described clearly how simultaneity in one system $\mathrm{S}$ became succession in another, $\mathrm{S}$, that was moving relative to S. He correctly identified the relation between simultaneity and the synchronization of clocks using light signals. (Poincaré had discussed these concepts with unambiguous clarity before 1905 [30]). He did not object to these conclusions in the theory of relativity. Bergson was simply insisting on the need to 'find to what extent it renounces intuition, to what extent it remains attached to it'. He concluded by raising the hope that the theory of relativity had nothing incompatible with the ideas of common sense. But he did not elaborate on what was required to achieve this compatibility.

Einstein's reply at Sorbonne was brief [1]. He summarized what he thought were the points raised by Bergson by saying,

The question is therefore the following: Is the time of the philosopher the same as the one of the physicist?

And finally answered,

There is not a time of philosophers; there is only a psychological time different from the time of the physicist.

The transcripts of this brief and restricted encounter are inadequate to see the really serious mature analysis by Bergson. For that one has to dwell into D-et-S. There, Bergson analysed Einstein's own example for establishing the relativity of simultaneity, and showed that the entire argument was inconsistent.

\subsection{Einstein's train and platform scenario}

We start with Einstein's own example, elaborated in his 'Relativity: The Special and General Theory' [19]. 


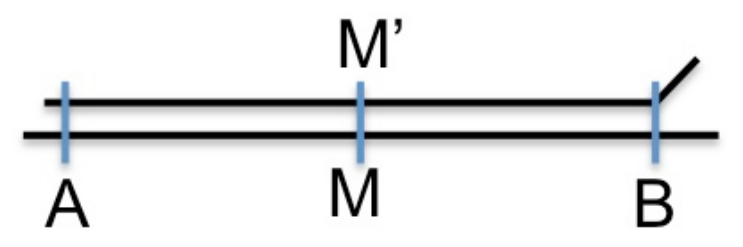

Figure 4: Einstein's example reference systems for discussing the relativity of simultaneity. The train and the railway platform are in relative motion, with 'midpoint' observers M' and $\mathrm{M}$.

We suppose a very long train travelling along the rails with the constant velocity $\mathrm{v}$ and in the direction indicated in the figure (4). People travelling in this train will with advantage use the train as a rigid reference-body (co-ordinate system); they regard all events in reference to the train. Then every event which takes place along the line also takes place at a particular point of the train. Also the definition of simultaneity can be given relative to the train in exactly the same way as with respect to the embankment. As a natural consequence, however, the following question arises:

Are two events (e.g. the two strokes of lightning A and B) which are simultaneous with reference to the railway embankment also simultaneous relatively to the train?

The problem is clearly stated. We will now try to answer the query in two steps; first, under the assumption that the relative velocity of the 'messenger waves' that carries the information of the events is Galilean, and second, that is an invariant, as Einstein postulated for light. Consider two observers $\mathrm{O}$ and $\mathrm{O}^{\prime}$ in relative motion. $\mathrm{O}$ is stationary and at the midpoint $\mathrm{M}$ relative to the sources of the Galilean waves, say sound from two alarm bells (see figure 5). Therefore, $\mathrm{O}$ hears the bells simultaneously, say at time $t=T_{0}$. Conversely, if $\mathrm{O}$ hears the bells simultaneously, then it is deduced that they were sounded at the same time $\left(t=T_{0}-d / c_{s}\right)$ at $\mathrm{A}$ and $\mathrm{B}$. The observer $\mathrm{O}^{\prime}$, who coincides with $\mathrm{O}$ at $\mathrm{M}^{\prime}=\mathrm{M}$ when the bells were sounded, is in motion towards $\mathrm{B}$ at the relative speed $v$. In his rest frame, the waves are arriving at relative speeds $c_{s}+v$ from $\mathrm{B}$ and $c_{s}-v$ from $\mathrm{A}$. Therefore, $\mathrm{O}$ ' will hear the bell from B first, at $t_{1}^{\prime}=L /\left(c_{s}+v\right)$ and that from A later, at $t_{2}^{\prime}=L /\left(c_{s}-v\right)$, and the events would be judged as not simultaneous. Thus, the unambiguous answer to Einstein's query for the case of Galilean waves 
is that different observers perceive the order of events differently, depending on their state of motion. Events that the stationary observer perceives as simultaneous will not be simultaneous for the observer in motion. Identical conclusion is reached from the rest frame of the stationary person as well; the moving person moves away from the midpoint and therefore the sounds of bells reach him in succession from the locations $B$ and $A$. There is no reciprocity.

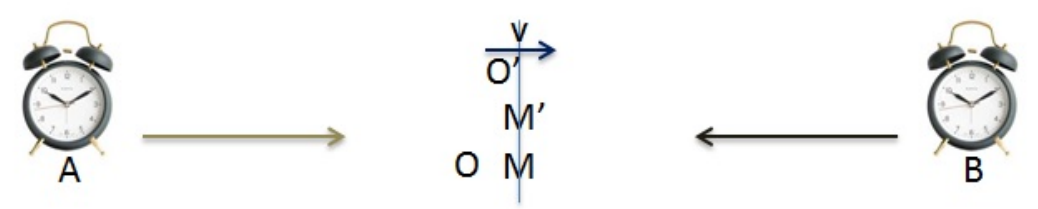

Figure 5: The situation regarding simultaneity with Galilean waves as the messenger of events that are spatially separated. The lack of absolute simultaneity is, however, only apparent because the Doppler effect.

We note an important additional point that is obvious in the case of sound. Not only that the observer O' perceives the bells in succession, he would also perceive them shifted relatively in pitch; the one from $\mathrm{B}$ at a higher pitch than the one from A, due to the Doppler effect. Hence, he has a measure of his real motion and velocity relative to air. Therefore, absolute simultaneity is recoverable when the messenger of the spatially separated events is Galilean.

Now we analyze the same situation under the assumption that the speed of light is the same in all inertial frames, independent of their velocity, as Einstein had assumed. Due to this cardinal difference from familiar Galilean waves like sound, we expect a different conclusion regarding simultaneity when light is the messenger, instead of sound. Then, we will examine the analysis and answers given by Einstein and Bergson. To avoid the prejudice of inadvertently choosing one frame over the other as privileged, we deal with the question rigorously from two identical reference systems, both of which are trains in relative motion, instead of a platform and a train.

In the rest frame of the train S', all its clocks read the same time and observer at M' at the midpoint is at rest. Relative to S', the train $S$ is moving. But that is irrelevant for what the observer of $S^{\prime}$ experiences in S'. After lightning strikes A' and B', light travels at equal speed from the equidistant points A' and B' towards M' (the relative velocity of the source 


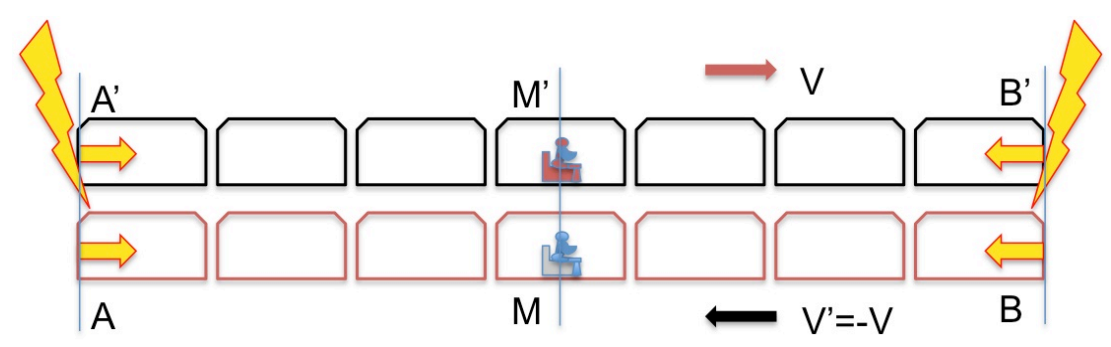

Figure 6: Reference frames $S$ and $S$ ' with relative motion at speed $v$. Lightning strikes the end points A and B (and A' and B') when the midpoints M-M' of systems coincide.

does not affect the relative velocity of light). Then, the light flashes will arrive simultaneously at M' and the observer perceives the events as simultaneous.

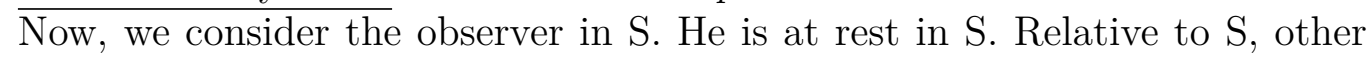
frames like $S^{\prime}$ are moving, but that is irrelevant for what the observer of $S$ experiences in S. Light pulses travel at equal speed from equidistant points $\mathrm{A}$ and $\mathrm{B}$ towards the midpoint $\mathrm{M}$. They will reach simultaneously at $\mathrm{M}$, and

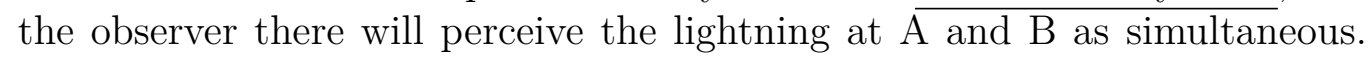
So, we conclude that the experiences of observers in $S$ and $S^{\prime}$, in their own rest frames, are identical, assuming the universal speed of light. Let us call this the unambiguous conclusion $\mathrm{U}$.

We note that if light propagated similar to sound and if its relative speed was not a universal constant, $c$ in one system (say in S') and unequal $(c \pm v)$ from $\mathrm{A}$ and $\mathrm{B}$ in $\mathrm{S}$, the events would have been seen as one after the other, in succession, in $\mathrm{S}$. This is the situation in Lorentz's ether relativity. In a frame immobile in the ether, light travels at identical speed in all directions, whereas in frames that are moving in ether, the speed of light is not isotropic. Thus, light pulses originated from the equidistant points A and B arrive one after the other. This can also be interpreted from the immobile (ether) frame with the same conclusion; as light travels at speed c, the frame that is moving relative to the ether is rushing towards one pulse and moving away from the other, hence the appearance of succession. The nature of propagation of the waves determines the nature of simultaneity.

What was Einstein's answer, which is also today's physicists' answer? Continuing with what Einstein wrote,

Are two events (e.g. the two strokes of lightning A and B) which are simultaneous with reference to the railway embankment also 
simultaneous relatively to the train? We shall show directly that the answer must be in the negative.

When we say that the lightning strokes A and B are simultaneous with respect to the embankment, we mean: the rays of light emitted at the places $\mathrm{A}$ and $\mathrm{B}$, where the lightning occurs, meet each other at the mid-point $\mathrm{M}$ of the length $\mathrm{A}-\mathrm{B}$ of the embankment. But the events $\mathrm{A}$ and $\mathrm{B}$ also correspond to positions $\mathrm{A}$ and $\mathrm{B}$ on the train. Let $\mathrm{M}$ ' be the mid-point of the distance $\mathrm{A}-\mathrm{B}$ on the travelling train. Just when the flashes of lightning occur (as judged from the embankment), this point M' naturally coincides with the point $M$, but it moves towards the right in the diagram with the velocity $\mathrm{v}$ of the train. If an observer sitting in the position $\mathrm{M}^{\prime}$ in the train did not possess this velocity, then he would remain permanently at $\mathrm{M}$, and the light rays emitted by the flashes of lightning $\mathrm{A}$ and $\mathrm{B}$ would reach him simultaneously, i.e. they would meet just where he is situated. Now in reality (considered with reference to the railway embankment) he is hastening towards the beam of light coming from B, whilst he is riding on ahead of the beam of light coming from A. Hence the observer will see the beam of light emitted from B earlier than he will see that emitted from A. Observers who take the railway train as their reference-body must therefore come to the conclusion that the lightning flash B took place earlier than the lightning flash A. We thus arrive at the important result:

Events which are simultaneous with reference to the embankment are not simultaneous with respect to the train, and vice versa (relativity of simultaneity).

Einstein's conclusion was identical to the one we arrived at in the case of Galilean waves (sound)! Decades of reading these passages by generations have not spotted why the conclusion with light ended up in preferential simultaneity of the lightning flashes for the observer on the embankment and events in succession for the observer in the train (as it would happen with sound waves) in a theory in which both observers are equivalent; they have the same speed of light, and either can consider himself as at rest and the other one as moving. Where is the gap of logic in Einstein's analysis that led to a different inference from the conclusion $U$ we arrived at earlier? If 
Einstein had started with the train as the reference, he would have reached the conclusion that the events were simultaneous in the train, but not on the embankment that moved relative to the immobile train. That is what follows from assuming the same relative velocity light in either frame. It is clear that Einstein would have been saved from this error of inadvertent Galilean preference for the embankment as 'really stationary' if he had considered two trains in relative motion. ${ }^{7}$ That the experience of simultaneity will be identical is clear from our discussion with the reference frames of two trains. A few like Bergson did find a problem with Einstein's logic and expressed disagreement.

What Einstein describes is not what the observer in the train experiences, but what the person on the platform, who considers $M$ ' as moving, confers on the moving observer as his experience. However, a crucial detail of physics is missing even in that; the train of thoughts of the observer on the platform should have moved further in Einstein's analysis as,

Now in reality (considered with reference to the railway embankment) he is hastening towards the beam of light coming from B, whilst he is riding on ahead of the beam of light coming from A. Hence the observer will see the beam of light emitted from B earlier than he will see that emitted from A. The light from $B$ will be more blue and the light from A more red, compared to the light pulses seen by the stationary observer, due to the Doppler effect...

Thus, even if one goes with the way Einstein analyses the scenario, the Doppler effect will allow the detection of true motion and then simultaneity can easily be regained by correcting for the shift. Einstein's imagined lack of simultaneity is Galilean and apparent, and not fundamental, because the observer who experiences the events in succession will also see the signals differing in their frequency content due to the Doppler shift ('imagined', because it is not consistent with the assumption of an invariant relative speed of light).

\footnotetext{
${ }^{7}$ This is not the only instance when Einstein's usually clear writing generated long lasting confusion, which was never properly clarified. The question whether a rotating disc has the ratio of its circumference to radius as $2 \pi$ or different due to length contraction, was answered by Einstein as $>2 \pi$ and by most others as $<2 \pi$. The issue is continued to be written about. What is your answer?
} 


\subsection{Simultaneity in D-et-S}

Armed with this clarity, we can now examine Bergson's analysis of simultaneity in D-et-S (Chapter 4). Bergson covers both physical and philosophical issues in his comments. We discuss here only the physical and logical aspects. He starts by quoting Einstein's scenario of the train and the platform and his conclusion of relativity of simultaneity verbatim (the passages quoted above). Then he writes,

This passage enables us to catch on the wing an ambiguity that has been the cause of a good many misunderstandings...we must not forget that the train and the track are in a state of reciprocal motion... Let us now emit our two flashes of lightning. The points from which they set out no more belong to the ground than to the train; the waves advance independently of the motion of their source.

It then becomes evident at once the two systems are interchangeable, and that exactly the same thing will occur at M' as at the corresponding point $\mathrm{M}$. If $\mathrm{M}$ is at the middle of $\mathrm{AB}$ and if it is at $M$ we perceive a simultaneity on the track, it is at $M$ ', the middle of B'A', we shall perceive this same simultaneity in the train...what is simultaneity with respect to the track is simultaneity with respect to the train.

So, Bergson reached the conclusion that contradicted Einstein's incorrect one. Remarkably, it is the same as our conclusion U. Bergson showed that the asymmetrical conclusion on simultaneity that Einstein arrived at was merely a result of the preferential choice of the frame of the platform as 'stationary' and that of the train as 'moving'. If we had started with the train as the rest frame and platform as moving, we would come to the opposite conclusion. Since both the frames are equivalent, their simultaneities are also identical. The irony of being misunderstood and judged wrong on this aspect by generations is indeed tragic. Tragic for both Bergson, and for generations of the entire physics community.

We have seen that on both counts of multitude of times and the relativity of simultaneity in Special Relativity, Bergson was on solid logical and technical ground, as it can be verified easily. We also stress again the important fact about the Doppler asymmetry, which is never brought up in such discussions. It is obvious that an observer M' 'hastening towards the beam of 


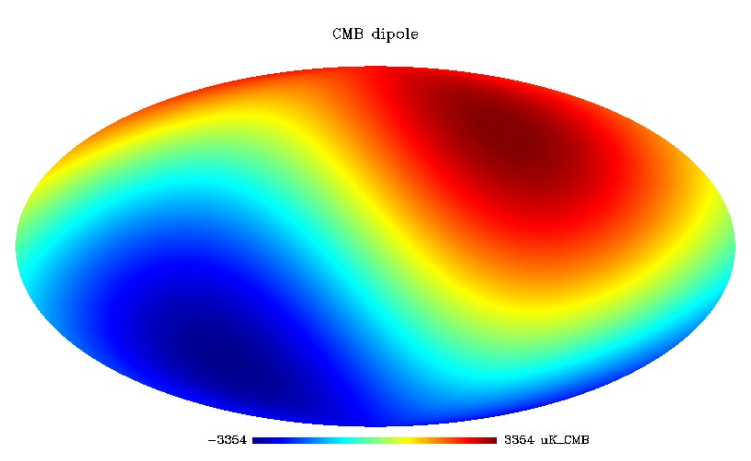

Figure 7: The dipole anisotropy in the temperature of the cosmic microwave background, as measured from the earth, indicating precisely the velocity of the earth relative to the isotropic universe. The average temperature $(\approx 2.73 \mathrm{~K})$ is Doppler shifted up (red in colour) by $v / c$ in the direction of motion and down, in the opposite direction. The precision of the measurements are now good enough to measure the velocity relative to the universal frame accurate to about a $\mathrm{m} / \mathrm{s}$ ! Seen as a cosmic clock, this is the same as the universal simultaneity appearing as a succession.

light coming from $\mathrm{B}$, whilst riding on ahead of the beam of light coming from A' will see light from B blue-shifted and that from A red-shifted, by approximately the fraction $\pm v / c$. Thus, experience of succession of the two events is associated with the Doppler asymmetry and unequal relative velocities of light. In modern times, a spectacular example is provided by what is called the 'dipole asymmetry' in the cosmic microwave background radiation, by which we know that we are moving in this universe at a velocity of about $369 \mathrm{~km} / \mathrm{s}$, accurate to a $\mathrm{m} / \mathrm{s}$.

\section{The Hadamard Catastrophe}

In one session at the Collège de France, a question was posed by Prof. Jacques Hadamard on one of the simplest solutions of Einstein's General Theory of Relativity, which is the theory of gravitational phenomena. Hadamard was the professor of celestial mechanics at the Collège de France. The solution in question was found by Karl Schwarzchild in 1916, within a year of the publication of Einstein's theory [31]. It represented the gravitational field outside a spherical mass, like the earth or the sun. The solution, however had a noticeable mathematical issue. It had a terms that could go to zero or infinity as the distance from the centre of the mass reached a critical value. 
Could it become infinite in some situation in nature, when mass is very high or when the object is very compact?

Hadamard's question was about what one calls a black hole and its event horizon in the modern terminology. Einstein was concerned about the issue. He called it the 'Hadamard Catastrophe'. The physics of stellar evolution was not known properly in 1922 and it was not decidable whether the embarrassing situation would occur in the case of sufficiently massive stars. S. Chandrasekhar's discovery of the continued gravitational collapse of stars beyond a critical mass was still about a decade away [32]. Einstein had done some calculations that indicated that the gravitational time dilation would become so large as to freeze time before any physical quantity becomes infinite. He wondered whether the 'energy of matter transforms into energy of space'. Einstein did not want to speculate further. And Hadamard was apparently satisfied that some other physical process would likely intervene before an infinity appeared in the horizon.

There is a description of the Schwarzchild solution that avoids the infinity, but not the freezing of time. Curiously, one of the participants in the Paris sessions, Painlevé, had already found its mathematical form in 1921 [33]. Painlevé's coordinates turn out to be just a Galilean transformation to the free-fall frames, similar to the one found by Langevin for rotating frames, discussed in the next section. Thus, the black hole solution in General Relativity is not dependent on Special Relativity and its features. Many conceptual problems related to the event horizon and time are discussed even more vigorously in the modern times, in the context of astrophysical as well as imaginary black holes.

\section{Sagnac's Proof for Ether}

There was one physicist in the audience at the Collège de France who could have contributed decisively to the debate about the nature of the theory of relativity, with his experimental results. However, Georges Sagnac's intervention was not effective and was not even mentioned by people like Nordmann. This detail is from the memoirs of the Nobel laureate Alfred Kastler, who was a first year student at the Ecole Normale Supérieure in 1922 [34]. As a student attending Langevin's lectures, he got access to the events at the Collège de France. He writes from the 'unforgettable memory of those sessions', 
Allow me to recall a memory from my youth: I was a first year student at the Ecole Normale Supérieure at Ulm street, when Paul Langevin had the bold idea in 1922 to invite Einstein in Paris. The undertaking at that time was not without risk because Einstein was then professor in Berlin and anti-German demonstrations could be feared. However all went fine. Einstein started to give a talk for a general audience about the idea of relativity in the packed big auditorium of the Collège de France...

There were dramatic moments too. For instance when old Sagnac, the inventor of the ingenious interferometer, gave vent to his anger against the theory of relativity, on which he put all the blame. The only way was to let the storm die down. ${ }^{8}$ And there were difficult moments too: when great mathematician Paul Painlevé talking about the adventure of the two friends the one who stayed in a place and the one who left by train and came back - refused obstinately to understand why the latter had remained younger than the first one. One must say that the trivial interpretation given about this effect is not satisfactory, because one forgets to mention that the traveller, while turning back, is subjected to a considerable acceleration, and that to be treated in depth, the problem must be studied from the point of view of general relativity.

There are references to two fundamental issues here. The one on the twin paradox, debated by Painlevé, we have already discussed. It is mentioned here again to stress the point that the paradox has no satisfactory solution, according to Kastler, within Special Relativity. His opinion was that the problem must be studied within the theory of General Relativity, and hence involves gravity, as Einstein had done. (This view has been dismissed by many modern authors, citing irrelevant reasons - it is unlikely that they have read Einstein's reasoning and the simple General Relativity treatment of this problem - see appendix I).

Let us turn to the other issue raised by Saganc that is extremely important

\footnotetext{
${ }^{8}$ This incident of 'violent intervention' is mentioned by the physicist and writer J-P. Pomey as well, who was present at the session (Les conférences d'Einstein au Collège de France, Le Producteur (1922) 8, 201-206). Despite its intensity, Sagnac's outburst was ignored and quickly forgotten.
} 
for the history of physics and for the physics of relativity. There is no detail on what exactly Sagnac said, but the context is obvious. Georges Sagnac was well known for his research in X-rays and optics. In 1913 he used a closedloop optical interferometer to measure the effect of rotation through the 'stationary ether'. He presented the positive results, 'the proof of the reality of the luminiferous aether by the experiment with a rotating interferometer', in several publications $[35,36]$. He considered his result as the decisive disproof of the etherless Special Theory of Relativity.

It is obvious that the import of Sagnac's results was largely ignored. That was the reason for the 'angry excitement of old Sagnac' that Kastler wrote about (Sagnac was only about 50 years old in 1922, but perhaps was perceived to be older. Details on Sagnac's scientific career and work was researched and published recently by O. Darrigol [37]).

A. A. Michelson had published a similar idea in 1904, to detect earth's rotational motion relative to the ether, but managed to do an experiment only in 1924, with H. G. Gale [14, 38, 39]. The idea of similar 'first order' experiments in the context of the ether dates back further; see the discussions by Oliver Lodge in 1893 and 1897 [40]. Michelson concluded from their heroic experiment, with a partially evacuated kilometer-size closed rectangular interferometer, that the slow rotation of the earth relative to the stationary ether was detected. As stated in the abstract of their paper, they found that the beam traversing the rectangle in the counter-clockwise direction was retarded (that is the direction of the rotation of the earth). Michelson was aware of Sagnac's experiment, but apparently believed that he could possibly get a different result because of the possibility that he massive earth might drag the ether with it, near its surface [41]. Somehow, Langevin and others, who commented extensively on Sagnac's experiment, have not analyzed the Michelson-Gale experiment explicitly.

Sagnac effect, as it is called now, is one of the most important technical tools to measure rotations optically, while being in the rotating frame. It is the basis of the fibre-optical gyro; one may call it the 'absolute rotation' detector. Conceptually, the Sagnac effect is in the class of the Foucault effect, the turning of the plane of a simple pendulum in a rotating frame like the earth.

The essence of Sagnac's experiment can be stated in a simple way, either from the rest frame of interferometer or from the 'ether frame' relative to which the interferometer rotates (Sagnac's own reasoning was more involved, and was tied to his own theory of the ether [37]). Imagine the ether 

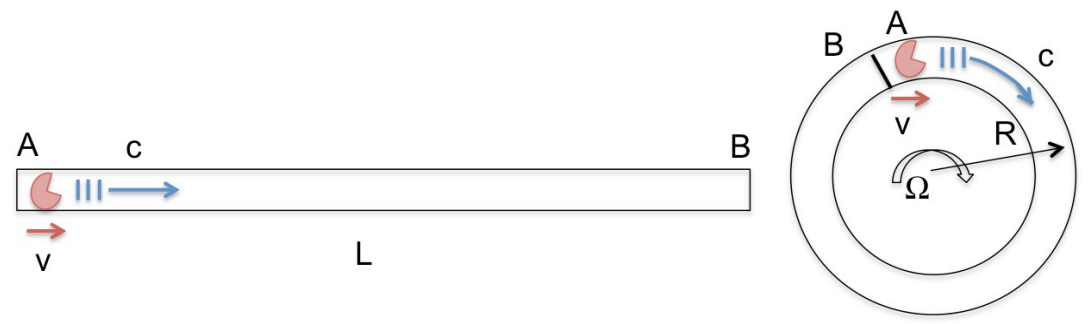

Figure 8: The equivalence of the relative durations the wave takes to propagate a relative distance $L$, while the frame is moving at the velocity $\mathrm{v}$, for the linear case (left) and for the looped circular case (right). Here, $2 \pi R=L$, and $R \Omega=v$. The experiment on the left is not feasible, because the waves would be far from the observer, at the end B, where another synchronized clock is required. The one on the right is the Sagnac experiment.

medium, relative to which light travels at velocity $c$, taking duration $T$ to travel a fixed distance $L$. If one moves in the direction of the light waves at velocity $v$, chasing light through the stationary ether, light waves will be moving at a lesser relative velocity, at $c-v$, just as the relative velocity of sound waves changes for an observer who moves through stationary air medium. Then the duration of propagation over the same distance will be longer. If we reverse the direction of the light waves, by reflection as in Michelson's experiment, the relative velocity increases, $c+v$, duration decreases and nearly cancels away the increase during the up-trip. However, instead of reflecting and reversing the path, if we arrange to loop the path, by identifying the end points of the total path, the frame is moving in the same direction through the medium anywhere along the path, and the cancellation is avoided! Thinking about the case of sound, one can see that this gives the same result as measuring the relative velocity along a straight path, $c-v$, if we rotate in one direction. If light is sent opposite to the rotation, the relative velocity is $c+v$. The two can be combined using two identical waves going clockwise and counter-clockwise, while the rotation is only in one sense; then the time taken to cover the same loop distance $L$ (in the rest frame of the observer) by one wave is different from the time taken by the other,

$$
\delta T=\frac{L}{c-v}-\frac{L}{c+v} \simeq \frac{2 L v}{c^{2}}
$$

When the light beams are combined, this difference manifests as the phase difference and the shift of interference fringes. (The situation is similar, and easy to understand, for sound waves in a similar device rotating relative to 
still air).

Note that we have not considered any frame outside the experimenter's rest frame, to derive this result. The simple result can be written in a more general form, with the same content. Since $L=2 \pi R$ and $v=\Omega R$ for a path looped into a circle, $\delta T=4 A \Omega / c^{2}$, where $A=\pi R^{2}$ is the 'area' of the interferometer.

The result can also be derived from the frame of the stationary medium, as usually done. Then, the speed of the waves is the same in both directions. So, as the waves progress, the interferometer is rotating and the end point is moving away from one wave and moving towards the other. ${ }^{9}$ Thus, one wave will take more time, and the other less, to reach the common end point that has moved a distance, $\delta L \simeq L v / c$ in the nominal round trip time of $L / c$. The total difference is $\delta T \simeq 2 L v / c^{2}$, same as the value found in the rest frame. This is the same as the splitting of simultaneity into succession due to the Galilean nature of the waves.

Unfortunately for Sagnac, his interpretation of the result of his experiment remained ignored, while he is celebrated now for inventing the 'Sagnac effect' (A prominent review paper on ring laser gyros mentions that 'Sagnac invented a rotation sensor' in 1913, without mentioning that it was an experiment that claimed the 'demonstration of the luminiferous ether'). The 'Sagnac term' is invoked to account for the motion of the frame in clock comparison experiments and in the analysis of time in GPS clocks. Michelson, though aware of Sagnac's work with the rotating interferometer, did not mention Sagnac's experiment in his 1927 monograph, 'Studies in Optics' [14]. However, a paper by L. Silberstein in 1921 on the propagation of light in rotating systems discussed both Sagnac's experiments and Michelson's proposed experiment, in the context of the ether and relativity [42]). The intuitive reasoning behind the experiments were similar. While Sagnac measured the rotation of a local frame relative to the ether, Michelson measured the rotation of the earth itself relative to the ether, according to their interpretations.

The analysis of Sagnac's experiment by Langevin (in 1921 and later in 1937) was perhaps the cause of the fading away of the significance of Sagnac's

\footnotetext{
${ }^{9}$ To use Einstein's expression, 'hastening towards the beam of light coming from B, whilst riding on ahead of the beam of light coming from A'; it is easy to see the similarity to the failure of simultaneity due to the Galilean nature of light.
} 
experiment for relativity, forever [43]. We mention this important technical remark right away. In that analysis, Langevin could hide (unintentionally, I suppose) a pure Galilean transformation in the language of metric in geometric General Relativity [44]. The true case is easily verified by simple inspection; Galilean transformation is

$$
t^{\prime}=t, x^{\prime}=x-v t
$$

This gives a transformed metrical structure from

$$
d s^{2}=-c^{2} d t^{2}+d x^{2} \Rightarrow-c^{2} d t^{2}\left(1-v^{2} / c^{2}\right)+2 v d x d t+d x^{2}
$$

If the straight path is looped around, as in the Sagnac device (as in the Fig. $7), x=R \phi$, where $\phi$ is the angle from one end point. Then the velocity is $v=R \dot{\phi}=R \Omega$, and $x^{\prime}=R \phi-R \Omega t$ is the Galilean transformation. Therefore,

$$
d s^{2}=-c^{2}\left(d t^{2}\right)\left(1-R^{2} \Omega^{2} / c^{2}\right)+2 R \Omega d \phi d t+d R^{2}+R^{2} d \phi^{2}
$$

So, we have the same linear Galilean transformation, now looped around in a mathematical bending. This is the Langevin metric, which is identical to the Galilean metric. This is so evidently not Special Relativity or General Relativity (the theory of gravity). A transformation that was consistent with Special Relativity would have been the one L. Thomas found in 1926 for atomic physics of electrons in orbits [45]; a set of successive Lorentz transformations in progressively changing directions is equivalent to a Lorentz transformation and a pure rotation, and not to a Galilean transformation. But then, the Sagnac effect disappears from the theory.

Therefore, any result that agrees with the Langevin metric is a direct proof of the Galilean nature of light and could be interpreted as the proof of existence of a privileged frame, like the ether. The language of the metric used in Langevin's analysis has mislead us to believe, naively, that the derivation is 'general relativistic'. Sagnac was right in his logic, for everybody to see. Thus, what Langevin did, and what people follow now, is Galilean relativity for the case of Sagnac's result, similar to what an ether relativist (LPTR) would do. The Galilean residual term is $L v / c^{2}$, as we already showed. In the unfortunate twists of history, the confused community psyche prevailed.

Though Alfred Kastler turned his academic career and attention to the interaction between atoms and light, eventually winning the Nobel prize in 1966 for the discovery of 'optical pumping', his intellectual interests spanned 
wider, including relativity, universe and gravity. (The laboratories of the Ecole Normale Supérieure, where many aspects of atom-light interactions are studied, is now called the Kastler-Brossel Laboratory (LKB), on the rue Lhomond, crossing the rue d'Ulm). In the Einstein birth centenary (1979) paper [34], where Kastler recounted the events of 1922, he explored Einstein's thoughts on the gravitational origin of inertia, and Ernst Mach's speculations [46] on the important topic. Kastler was obviously not aware of the detailed published work of Dennis Sciama [47] in the 1950s, 'on the origin of inertia'; Kastler estimated the gravitational potential of the matter in the universe and showed that it was of the right order of magnitude to explain inertia as due to the gravitational interaction with the rest of the universe. ${ }^{10}$ Where Sciama and Kastler stopped short, is indeed the door to the true relativity!

\section{A New Beginning}

We have reached the end of our impressionist glimpses of Einstein's visit to Paris in 1922. While focussing on the physics of the issues raised by Bergson in the context of Einstein's theory, we examined several connected aspects, in particular Einstein's own analysis of simultaneity and the twin paradox, Becquerel's confusion regarding clocks in Special Relativity, and Sagnac's 1913 experiment claiming the detection of ether. A significant revelation shown transparently is the verity of Bergson's critical view on relativity of simultaneity in Einstein's theory. The implied Galilean nature of light then brings forth Henri Poincaré's clear interpretations of Lorentz's local time, clock synchronization etc., in the context of the ether and relativity. The missing knowledge then and now was the role of the gravity of the matter-energy in the universe, which made brief strokes in the minds of a few, like Sciama and Kastler. But nobody took it all the way along its logical path to realize the profound fact that the old ether is to be replaced by the gravity of the matter-energy of the Universe, which defines the universal reference frame and determines the nature of dynamics. All the fundamen-

\footnotetext{
${ }^{10}$ During my visits to the Kastler-Brossel Laboratory (after 1997), in the context of laser cooling experiments on Helium, I remained unaware of Alfred Kastler's interest in Mach's principle and gravity, until recently when Serge Reynaud of LKB mentioned it and gave me the relevant papers. The link of one of those papers to the 1922 event was a pleasant coincidence.
} 
tal theories of physics were concretised before the observational results that indicated the vast universe and its gravity started to become available [48]. Current theories that do not incorporate this enormous gravity are flawed. The theory of relativity that is consistent with such a universe needs a drastic and fundamental change of the paradigm of relativity. I call this theory Cosmic Relativity [44, 49]. Its core predictions [44, 51] have been experimentally verified.

When the General Theory of Relativity was formulated as the relativistic theory of gravity, the basis was the special theory, set in empty space. This was dictated by the empirical knowledge about 'space' at that time. The single most important empirical gap that existed in the early 1920 s was the lack of knowledge about our Universe. We had only an imagined universe and its cosmology then, with very little matter around in the very distant stars and a few nebulae, apart from the globes of the solar system. Though the distant stars could provide a stable reference frame to measure our motion, there were no reliable physical arguments to give the special status to that frame as a determining, master reference frame. Therefore, both the Special Theory and the General Theory of Relativity were explicitly formulated with the empty space as their background. That was the significant deviation from the Lorentz-Poincaré theory, which had the ether as the special reference frame. Since empty space remains empty and the same for every observer in whatever state of motion, all observers were equivalent in Einstein's theory, with equal status to claim the state of rest.

Einstein applied his new theory of General Relativity to describe the universe and the gravity of its matter content already in 1917. By 1922, the new large telescopes were starting to see much deeper into the sky, opening up the vast extra galactic universe. In 1922, Alexander Friedmann published his ideas on the expanding universe with matter [52]. Yet, Einstein, or anybody else, did not realize that the kinematic phenomena that happen in this universe was happening in the large gravitational potentials of all cosmic matter and hence, not in empty Minkowski space as Special Relativity assumed. This was not surprising in the early days of theoretical cosmology, when Einstein himself thought that the extent of the universe is not much larger than the size of the galaxy. But, it was indeed logical inertia to remain oblivious to the physical implications of the vast matter filled universe that emerged later. 


\section{Inertial forces: Newton, Mach and Einstein}

There was one unresolved issue though that troubled Einstein, and the problem goes back all the way to Newton. That had to do with the appearance of new forces without any sources when the motion is not uniform. For example, forces are felt in an accelerating mobile, or centrifugal forces are felt by an observer in a rotating frame. For Newton, this was evidence for the existence of 'absolute space', but he could not point to it. Newton opted for an indirect reality of Space and Time, independent of matter, with their own primordial existence and properties. Ernest Mach, much later, criticized Newton for positing a non-tangible absolute space and suggested that the inertial forces (proportional to the mass) might be due to some interaction with the rest of the matter in the universe [46]. ${ }^{11}$ Einstein sidelined the issue in Special Relativity by considering only inertial frames that do not accelerate or rotate. But, he had to go beyond this restriction to formulate the general theory, which was a theory of gravitation. Einstein had enthusiastically sought support in Mach's conjecture linking inertia and the matter in the universe. He even elevated the conjecture to status of a Principle. Yet, Mach's principle was left out in the final theory. There, aided by his brilliant insight, and noticing that the inertial forces were proportional to the mass just as gravity was, he postulated that the two were indistinguishable - a new principle of relativity, which is known as Einstein's Equivalence Principle. This was a second time Einstein went ahead converting a problem to be solved into a postulate. Thus, he sidelined the issue again, after struggling and failing to incorporate Mach's idea into his new theory. He retained the reality of the empty space, devoid of 'stars and the rest of the universe', as the inert background for his general theory as well. Einstein was aware that his theory had some results that kept suggesting to listen to Mach, but with the equality of all observers and consequent denial of a privileged frame capable of 'influence by interaction', Mach was definitely out. Mach's stand on the inertial forces was not explicitly discussed during the 1922 visit, though Mach's philosophical stand on certain issues did figure in the discussion. Mach was known to be critical of the theoretical structure of

\footnotetext{
${ }^{11}$ Mach was discussing the origin of the inertial forces which could be interpreted as the origin of inertia, but it should not be misunderstood as the problem of the origin of mass, the charge of gravity. Also, gravity was not invoked in Mach's proposal presumably because it was known that the weak gravity couldn't generate sufficient forces with the amount of distant matter known in the cosmology of the 19th century.
} 
General Relativity, and Einstein thought that was 'because he was old' [2]. In any case, relativistic physics and the theory of gravity inherited Newton's non-tangible space and time, stripped of their absolute character and superficially fused into a space-time with a hidden imaginary character to time (that is, when we describe space and time together, 'time' needs a multiplier $\sqrt{-1}$ ). Bergson commented extensively on this peculiar spatialization of time in D-et-S, but we do not discuss this aspect in this article.

\section{Going beyond: Cosmic Relativity}

The guiding light dawned slowly, after 1928, not too late for a rethinking on these issues. It was the new observational cosmology of the extra-galactic universe, after Hubble's discovery of the recessing galaxies [48]. The universe that emerged was really vast, with enough matter and mass to take Mach seriously, even with a feeble interaction between matter. And gravity was a well understood interaction. Unfortunately, neither Einstein nor anybody else retraced the path of rigorous physical logic with this crucial knowledge; the theories of relativity continue to assume that our physical space is the non-existent empty space, and our time has no relation to the cosmic matter. It is as if the standard physics of today considers the universe we see as a huge make believe film set with virtual reality galaxies without gravitating matter; the theories continue in their course set definitely in the 1920s. In reality, there is one master frame with its material reference markers as the galaxies, and one universal time, given by the expanding universe and its evolutionary clock (also termed 'absolute time', but only in the sense of being defined by the single master reference frame of the material universe. 'Universal time' is the appropriate term, to avoid any confusion. This is distinctly different from Newton's ideal and metaphysical absolute time).

The old ether was stationary and without evolution, whereas the real universe is evolving, as evident in its expansion. Thus, the evolving universe defines a universal time, which the ether was devoid of. The gravity of all the matter-energy in the universe physically determines the possibilities of relativistic dynamics. When this reality is acknowledged, one gets a new theory of relativity, incorporating Mach's ideas, and without inconsistencies. I have named this theory appropriately as Cosmic Relativity; it retains naturally all the empirically correct aspects known in relativistic physics, but it is distinctly different from Einstein's theories [49, 50]. In Cosmic Relativity, dynamics and the inertial forces are derived from the gravitational influence 
of the cosmic matter. The Principle of Relativity follows from the approximate large scale homogeneity and isotropy of matter-energy in the universe. Motional modifications of duration and length are really gravitational modifications, because of the modified gravitational interaction due to motion. The propagation of light (and gravitational waves) is Galilean, controlled by the gravity of the universe (the gravitational potential acts like an effective universal refractive index to limit the velocities of all propagating waves). Einstein's equations for gravity are modified by including the cosmic gravity and the master frame as part of the equation itself. Then, Mach is naturally integrated with Galileo, Newton, Lorentz and Poincaré, and Einstein, retaining only the consistent elements that constitute a satisfactory completion.

Cosmic Relativity demands and predicts that the relative velocity of light is Galilean. ${ }^{12}$ An empirically rigorous experiment to test whether the one-way velocity of light, relative to inertially moving reference frames, is Galilean or Einsteinian needed innovations that avoided two spatially separated clocks and their synchronization, yet keeping the motion inertial. When this was achieved in our laboratory at the Tata Institute in 2005, the truth about the propagation of light was transparently visible; light, like waves of sound, is Galilean $[44,51]$. With new technology and lasers, the experimental task is much more simpler than what Michelson and Sagnac had to tackle. Thus, verifiable experimental results vindicate the logical proof we discussed, that the postulate of invariant velocity of light is inconsistent.

Cosmic Relativity firmly and surely brings back a real master frame that determines relativity and dynamics - the universe with its matter-energy and its gravity, in place of the old ether. Time now has a universal reference. But, there is real gravitational modification of duration in frames moving relative to the master frame, apparently shattering Bergson's hope for the single universal time. Is it possible to rebuild Bergsonian philosophy with its universal time afresh from this new reality?

\section{Resurrection of Bergson's Philosophy of Time}

We conclude the article with a new insight on Bergson's philosophy of time, with its basis on the notion of a universal time and the implied ab-

\footnotetext{
${ }^{12}$ This was the earliest prediction of the theory, even before many aspects of the theory were well understood. Galilean propagation of light is the logical consequence of a privileged frame.
} 
solute simultaneity. The reason Bergson ventured to study the theories of relativity was to understand the conflict between his philosophy and the physical theories, with their multitude of times and the relativity of simultaneity. In Lorentz's theory with the insensible ether, there was the direct and irreconcilable conflict; each observer had his own real time that depended on the speed relative to the ether, but there was no way to sense the state of motion. Bergson's hope was to find harmony with Einstein's theory, with its equivalence of all observers in motion and at rest, allowing the interpretation that the multitude of times was merely in the mathematical content of the theory without real physical manifestations. The empirical situation eventually proved otherwise, showing the reality of velocity dependent multitude of durations. But, we have seen that the real reason for the motional modification of duration could be found in the gravity of the matter-energy in the universe. This is consistent with both the Galilean nature of light and the existence of a privileged master frame. The amazing fact is that this frame, identified as the matter-filled universe, has both the universal time and the physical multitude of times, coexisting consistently. This is because unlike the situation in invisible and static ether, those who move have the correct measure of their motion with the cosmic matter and radiation as the markers of real rest, in the slowly expanding dynamic universe. The crucial physical distinction to note is that while the absolute nature of the old ether is truly insensible, the absoluteness of the material universe in time and space is real and easily observable, with its gravity manifest as the verifiable reason behind relativistic effects.

We can now assess the compatibility of Bergson's preferred view on time, duration and simultaneity, with the factual relativity and its theory, based on cosmic gravity (cosmic relativity). The modification of durations of a clock in motion is real, and therefore there are a multitude of real times. Similarly, simultaneity changes to succession for observers in motion, being linked to the Galilean nature of the relative velocity of light. Therefore, one might think that the Bergsonian philosophy of duration and simultaneity that needed a universal time and an absolute division between simultaneity and succession would crumble, as it would happen in the Lorentz-Poincaré 'half-relativity'. However, this judgement is premature. Since the factual relativity is entirely based on the gravity of matter-energy in the universe, there is a tangible privileged frame, relative to which all motion is felt and measured. No one in real motion can claim a state of rest because that will be in conflict with what he measures in his external world filled with matter 
and radiation, which are real material markers. This is a profound difference compared to ether relativity, in which the velocity of motion could not be detected by any means. In new relativity, the modification of the duration is related to the real measurable velocity relative to the cosmic matter (section 5.2). Therefore, an observer always has the access to both his proper time, measured by his clocks, and the universal absolute time measured by the clocks at relative rest to cosmic matter! This universal time, the same time everywhere in the universe, is operationally equivalent to the temperature of the cosmic microwave background radiation in the expanding universe. In fact, theoretical cosmology always worked with such a universal time, akin to Bergson's time, but never recognized that the physics done in this same and only universe has such a universal time. ${ }^{13}$

Now we can see that the notion of simultaneity of spatially separated events is also absolute, because these events are tagged to their local time which in turn is the single universal cosmic time. The succession experienced in perception is mere appearance, exactly as the change of simultaneity of sound of bells for one at rest into succession for another who is moving toward one of the bells. Since the velocity is known, simultaneity is regained, just as the universal time is regained.

Thus, we have a surprise end to our discussion. The postulated equivalence of the mobile and the immobile in the prevailing theory is incorrect. There is a universal time and a well defined reference for real motion. Physical theory of relativity, with its multitude of times and velocity dependent modification of physical quantities, nevertheless maintains harmony with the Bergsonian notions of a universal time and simultaneity. With the universal time regained, Bergson's philosophy remains intact. We are able to resurrect and complete Bergson's program in the philosophy of time, of finding compatibility with the notions of time and duration in relativistic physics [53]. Then, the time of the physicist and the time of the philosopher, and indeed the time of common intuition, all have the same basis and coexist without any logical or conceptual conflicts.

\footnotetext{
${ }^{13} \mathrm{~B}$. Latour has commented on the fact that Bergson stressed the 'cosmological import' of his notion of space and time and on the need to find consistency between subjective time and the time of physics (ref. [5]). I am arguing that the physical universe provides this consistency naturally through its physical effects that can be experienced.
} 


\section{Acknowledgements}

Many sections of this article were refined with the help of Martine Armand, whose translations and readings, while appreciating the angel's share, were important in going into details of the discussions and academic interaction during the 1922 visit. Continued help on many occasions from Michèle Leduc, my academic host for several years at the Kastler-Brossel Laboratory of the Ecole Normale Supérieure (LKB, ENS, Paris), was always a supporting factor. I am thankful to Serge Reynaud of LKB, ENS, who alerted me to Alfred Kastler's long term interest in Mach's hypothesis and provided the relevant articles.

\section{Appendices: Background to This Section}

In the main text we have discussed how the position taken by Einstein and his followers was not fully justified. We have already proved transparently how Einstein's analysis of the central aspect of simultaneity relative to two frames in relative motion was in conflict with his own postulate of the invariance of the velocity of light, confirming Bergson's comments on the issue.

The crucial insight that makes a drastic revision in our understanding of the theory of relativity is the definite knowledge of our universe, which was lacking in 1905-1915, or even in 1922. Thus Einstein's theories of relativity are built on the explicit and necessary assumption that space is empty devoid of any matter in general, like Newton's space. With no matter or material markers, empty space remains isotropic to an observer who moves in space. That is how Special Relativity has the invariance for the velocity of light and General Relativity has the general coordinate invariance. In the present paradigm, the universe is treated as just one of the many possible solutions of the Einstein's equations of gravity in General Relativity, and not as an a priori premise for all physical phenomena.

But this is obviously deeply flawed. The theories and equations of physics are operative in this universe, in the presence of all the matter-energy and its gravity. All tests of relativity and indeed all physical processes and dynamics happen in the presence of this gravity, but this presence is not part of the present theories! Even a cursory logical inspection reveals that we are seriously off the track and the correct theory should acknowledge and incorporate the privileged frame of the matter-marked universe, and a universal 
time in the ever expanding universe (also available as the monotonically decreasing temperature of the cosmic radiation background). The observer in real motion relative to this matter experiences a different world and physics compared to an observer who is at rest relative to this matter, due to the difference in the gravitational potentials in these two situations. This physical fact should be the basis of the correct theory of relativity, as achieved in the theory of 'Cosmic Relativity' [44, 49]. This unified theory of relativity and dynamics replaces the prevailing theory, and with that Einstein's General Relativity also is modified to include the gravity of the universe as part of the fundamental equation itself. There is no general coordinate invariance and the world of physics is factually Galilean and Machian, with all the empirically seen relativistic effects correctly predicated as gravitational effects of the matter in the universe.

We examine the empirically verifiable physical links between duration, motion and gravity in the appendices. In particular, I present Einstein' own resolution, in 1918, of the twin paradox [23], which should be an eye opener to those who are unfamiliar with that paper.

\section{Appendix I:}

\section{The Clocks of Twins and the Triplets}

The traditional debate regarding the 'relativistic twins' was about who ages more, Pierre or Paul. We know empirically that some transported clocks, like Paul, age less. We also know that some transported clocks, age more when they come back after their trip! A clock that is inertially transported, after synchronizing, from Mumbai to Paris by surface transport will actually age faster, and not slower [28]. To check this, one can accumulate the durations by transporting it further across the Atlantic and Pacific oceans, and back to Mumbai. The travelled clock would be about 100 ns older than the stationary clock in Mumbai, which never left the room. The reason for this cannot be found within Special Relativity. But in a theory with an absolute master frame, this is easy to understand, The 'frame at rest', in which Pierre resides, is really moving relative to the master frame and we should use that velocity to calculate the total real time dilation, and not the relative velocities of Special Relativity. However, this is not what we want to discuss here, because the experimental results are known [50], and such a calculation can be done by anybody to verify the facts. 
We go back to the question addressed by Bergson: how does one decide that one of the travellers ages less in the observer-symmetric Special Theory of Relativity (STR)? First, suppose one knew about only STR. Now we have three characters of the same age, Pierre, Paul and Pauline. Paul and Pauline are in their spaceships. Pierre, who is on a large space station, perhaps like a planet, is moving away relative to the other two. The large velocity of separation is $v$. Their clocks are synchronized before they separated. Who ages faster? We see that we are unable to answer this question, because we formulated the question correctly! Try answering it, in place of Becquerel and the many Einsteinian relativists through times, and we are immediately stuck, because Special Relativity is a symmetric theory. Paul and Pauline think that Pierre should age slower because they see his moving away, while they are at rest in their spaceships. Pierre thinks that he should age faster than Paul and Pauline who are moving away together. In a theory with a master frame, like the universe and its matter, there is no confusion. Those who move relative to the master frame, age slower. But in STR, at the end of half the story, a few years of separation, there is no definite answer to our question. Then the adherent of STR listening to the paradox fables would ask, "then what happened?", looking to break the symmetry.

Here, Bergson's questions as a philosopher, also reflecting common intuition, take relevance. Shouldn't a theory be able to give the answer at all times? Why does the theory need to know what happens in the scenario later, to say something about the lived durations? Surely, one of the clocks should be slower than the other, if such a physical effect is really there. Which one?

At least one fact is clear - Paul and Pauline, being side by side, age the same way, with their clocks remaining synchronized. The clocks of the triplets have some readings locally, and whatever they are, a clock's reading in one spacecraft cannot be changed by firing a rocket in another spacecraft far away. Nobody would agree to 'spooky action at a distance' in relativity's clocks. Since this is a very important point that is neglected in debates, let us state it clearly. Relative time is the difference in the readings of time in two distinct identical clocks; one quantity defined by two clocks. Therefore, relative duration can change only by changing one or both the readings. If acceleration has any role in changing the proper time, it can only affect the clock that is accelerated, a conclusion everyone would agree with. Hence, if two clocks were synchronized at some stage, after which they were in relative inertial motion, they would be completely equivalent is Special Relativity, till one of them is accelerated. When such an asymmetry occurs, only the clock 
that is accelerated can have a new physical effect altering its readings. So, there are three phases for the rest frame of the accelerated clock; two identical inertial stages of the round trip, during which it is reciprocally equivalent to the unaccelerated clock, and one intermediate stage of short acceleration for which we have to calculate the time dilation of that particular clock with an appropriate theory. At all times, the clock is at rest relative to itself.

Pauline decides to fire a rocket thruster briefly and arranges her flight path to be directed back to join Pierre. So, if at all there is some change to the clocks as a result of Pauline's action, that must affect only the reading of Pauline's clocks, and not Paul's or Pierre's. She is still near Paul, since she needed to fire the rocket very briefly; then there is only a slight difference between her clocks and Paul's, perhaps a few hours. Of course, nothing would have changed unusually in Pierre's clocks, while she fired her rocket. Since Paul and Pauline decided, using STR, that Pierre was ageing slower and must be younger by several months by then, that conclusion doesn't change by this difference of a few hours between the clocks carried by Paul and Pauline. Pierre, however, continues to think that the other two must be ageing slower. He also knows that there will only be a slight difference between their clocks if one of them fires a rocket briefly. Who really ages more and by how much? There is still no answer in STR.

That is why Einstein brought in the General Theory of Relativity and gravitation to break the symmetry and try to solve the puzzle [23] (commented as necessary by Nordmann and Kastler in their notes on the 1922 visit). He admitted that there could not be differential ageing in the symmetrical theory of STR. Then the differential ageing must be due to some other reason, to be handled by another theory.

Einstein's own resolution of the twin paradox

The paper that Einstein wrote in 1918 to explain his resolution of the twin paradox is important for both physics and the history of physics. Not many physicists are aware of the paper, as evident from their continued use of incorrect arguments for the resolution. Here is Einstein's simple General Relativity argument, which he thought enabled him to 'extricate skillfully' from the paradox eventually [23, 27]. First, he admitted that the time dilation in STR, while the clocks were in uniform relative motion, was symmetric. So, Pierre reckons that Pauline is younger by the factor $\gamma=1 /\left(1-v^{2} / c^{2}\right)^{1 / 2}$ and Pauline and Paul would claim that Pierre is younger by the same factor. If the relative velocity is not very high $(v<0.1 c)$, we may write the time dilation 
during the total flight duration $2 T$ as $\delta T \simeq T v^{2} / c^{2}$ (the sign is relative to the clock in the rest frame, indicating that the rest frame clock is faster). While Pauline fires the rocket to reverse the trip and join Pierre, her deceleration is $a$, for a duration $t$, such that her relative velocity reverses, $a t=2 v$. In Pierre's frame, that doesn't make much difference to her ageing, if $t$ is a relatively short duration. However, in Pauline's rest frame, she is not moving, but just experiencing a uniform gravitational field $g=-a$, extending over all space, according to Einstein Equivalence Principle. Then there should be a relative gravitational time dilation of General Relativity between the clocks separated by the distance $L=v T$ equal to $\Delta t=t g L / c^{2}=-t a L / c^{2}=-2 v L / c^{2}=$ $-2 T v^{2} / c^{2}$. We note the important feature that the amount of gravitational time dilation is independent of the duration of acceleration $t$. This is double the special relativistic time dilation and of opposite sign, Pauline's clock running slower than Pierre's, in her frame. The total time dilation is hence $\delta T+\Delta t=-T v^{2} / c^{2}$, which is the same conclusion as Pierre's; Pauline's clocks are 'younger' when they meet again.

That is how Einstein solved it. Or, thought he solved it, and was confident enough to write a paper describing the solution, with explicit statements that the time dilations in the symmetric Special Relativity were symmetric [23], a stand similar to that of Bergson! This position contradicts most texts on the theory. ${ }^{14}$

In Einstein's paper, the asymmetry of the acceleration and equivalent gravity were handled by the equivalence principle and General Relativity. But, he was not attentive enough, in details. There are two clocks in the problem and the formula is for the relative time. We just found that Pauline's clock was not affected by her brief deceleration, as she verified by comparing with the nearby Paul. Her firing the rocket could not have affected the clocks with Pierre, who was $L$ distance away from her! So, there must be something seriously missing in Einstein's solution. Even a layman can note that the entire argument collapses if Pauline chooses to 'arrest' or 'freeze' the clock during the brief period of noninertial motion. Then her clock cannot

\footnotetext{
${ }^{14}$ Einstein's close friend, Max Born, wrote in his textbook, Einstein's Theory of Relativity (p. 284), "Thus the clock paradox is due to a false application of the special theory of relativity to a case in which the general theory should be applied." However, he chose to claim that the resolution was fully within special relativity when the twin paradox controversy was discussed in the pages of Nature, in the 1960s (M. Born, Nature 197, 1287 (1963)).
} 
suffer any time dilation! Besides, take a look at the actual numbers in an example, within the approximation he used. If $a \simeq 10^{2} \mathrm{~m} / \mathrm{s}$ for two days, and $L \simeq 10^{15} \mathrm{~m}$, after a year's travel at $v \simeq 0.1 c$, all small compared to the numbers usually used in the discussion of the paradox, then $a L>c^{2}$; clearly the formula for the gravitational time dilation breaks down. Further, the twin paradox arises the same way even in a scenario where the clock readings are transferred between inertial frames (between two spaceships travelling in opposite directions) without any physical accelerations. The physical clocks or the observers are not transferred or accelerated; only the information on time is transferred from the frame moving at the velocity $\vec{v}$ to another that is moving at the velocity $-\vec{v}$. Then there is no equivalent gravity to induce Einstein's gravitational time dilation. Yet there is asymmetric relative time dilation. So, Einstein's resolution is not general enough to resolve the paradox, and cannot be the genuine resolution [27].

However, if one rejects Einstein's resolution, by claiming an entirely special relativistic resolution, one is rejecting Einstein's Equivalence Principle and General Relativity. The relative time dilation of clocks in an accelerated frame is a prediction of General Relativity. That is the serious dilemma one has to face. As indicated earlier, the correct solution is in the paradigm of Cosmic Relativity, in which the Einstein equivalence between clocks in an accelerated frame and in a uniform gravitational field is not valid [50]. All motional time dilations are factually gravitational time dilations in the velocity-dependent gravitational potential of the matter in the universe. Acceleration is not important in the time dilation problem. It is significant that the relative time dilation of clocks in an accelerated frame is not yet tested.

\section{Appendix II:}

\section{A Lesson on Time From Birds Flying Backwards}

We can briefly come back to the theme of the flock of birds seemingly going backwards, taking it as a metaphor. In Einstein's theory, relativistic physical effects depend on the relative velocity as the sole parameter. For two frames in inertial motion, only their velocity of separation, or the relative velocity, makes physical sense in empty space. However, when there is a privileged frame and universal markers in space, the situation is different. In the old ether relativity, it was the electromagnetic properties of the ether that were meant to induce motional physical effects. In Cosmic Relativity, it is the gravity of cosmic matter that is the source of all relativistic effects. In 
such theories, it is the absolute velocity - the velocity relative to the master frame - that determines the magnitude of the physical effect.

Consider an inertial reference frame from which Pierre is watching the world. Paul is moving fast to his left, and so is a flock of birds, only slower. The birds' timing and synchronization is admirable. Pierre concludes that Paul's clocks are slower than his. Pierre also estimates that the faster Paul would age slower than the birds. If they continue moving like that, birds should age more than Paul.

Paul greets Pierre as they pass by. Paul estimates that Pierre's clock is running slower than his. Then he sees the flock of birds, flying backwards. Being an adherent of Einstein's theory, he reckons that birds in relative inertial motion have their life and internal clocks running slower than his. If they continue their flights, the birds should maintain their slower ageing.

So, Einstein's theory does not give any weight to the logical inference that Paul is indeed moving faster than the birds and therefore one expects larger motional time dilation in Paul's clocks. As we emphasized earlier, relative time is the difference between the readings of two independent clocks, $t_{1}-t_{2}$. A complete physical theory should be able to provide an expression for the modification of the rate of the individual clock, $t_{1}$ or $t_{2}$, etc., from which one can calculate the relative time dilation. This is much like the spatial separation or the distance between two material points. The change in distance is effected by independent changes in either position.

This by itself does not reveal any inconsistency in Einstein's theory in the most common situations because an actual empirical comparison requires bringing the clocks together. Then the usual discussions reduce to a twoway transport of some clocks. However, think of Paul and the birds in their continued flight. After a very long duration, Paul encounters the same flock again, having gone around the globe in the long trip. Now he can compare the clocks; to his shock he would find that his expectation from Einstein's theory went wrong; the birds who were seen in relative motion actually aged faster! Special relativity is not adequate to explain the physical situation.

The correct physics of motional time dilation of clocks (as in GPS) is to be found outside Einstein's theory. I assert from Cosmic Relativity that the correct and most accurate expressions for the relative time (difference of 
elapsed durations) of two clocks is

$$
\begin{aligned}
\Delta T / T & =\sqrt{1-v^{2} / c^{2}}-\sqrt{1-u^{2} / c^{2}} \\
& \simeq-\left(v^{2} / 2 c^{2}-u^{2} / 2 c^{2}\right)=-\frac{(v-u)(v+u)}{2 c^{2}}=\frac{(u-v)(v+u)}{2 c^{2}} \\
& =-\frac{(v-u)(v-u+2 u)}{2 c^{2}}=\frac{(u-v)(u-v+2 v)}{2 c^{2}} \\
& =-\frac{v_{r e l}^{2}}{2 c^{2}}-\frac{v_{r e l} u}{c^{2}}=\frac{v_{r e l}^{2}}{2 c^{2}}-\frac{v_{r e l} v}{c^{2}}
\end{aligned}
$$

Here, the velocities $v$ and $u$ are relative to the cosmic frame, and $v_{\text {rel }}=$ $(v-u)=-(u-v)$. The asymmetry between the frames is explicit. When the velocities are not uniform, the infinitesimal durations can be accumulated (integrated) to get the total duration.

Positioning algorithms for the motional relativistic corrections in the Global Navigational Satellite Systems (GNSS) like the GPS were designed assuming the STR axiom of the invariant relative velocity of light. Light signals (radio-frequency) are used for the time transfer. Hence, from the measured difference of the clock times at the satellite and the receiver, $\Delta t=t_{s}-t_{r}$, the distance to a satellite was to be estimated as $d_{s}=c \Delta t$. In contrast, in a theory with the universe as the master determining frame, the distance would be calculated with the Galilean relative velocity as

$$
d_{s}=\left(c-\vec{c} \cdot \overrightarrow{v_{r}} / c\right) \Delta t \simeq c \Delta t-\overrightarrow{d_{s}} \cdot \overrightarrow{v_{r}} / c
$$

This is the equation that is factually used in GPS, and not the STR formula $d_{s}=c \Delta t$ ! Ironically, the correction is called the 'Sagnac term'.

Similarly, the second order clock correction used in GPS relies on the formula (6) and not on the special relativistic formula $-v_{r e l}^{2} / 2 c^{2}$. The 'absolute velocity' of the receiver explicitly appears though it is at rest in its frame. GNSS provide ample proof for Cosmic Relativity based on gravity of the matter-energy in the universe.

\section{Appendix III:}

\section{Lorentz's Local Time and the New Universal Time}

We are familiar with the local time set according to the celestial solar clock. This helps in adjusting the time of the day to the presence of the sun at each locality on the rotating earth. But, this has no deep physical 
significance. We do not infer the velocity of an aircraft using the difference in local times at the start and the end of the journey. Everybody understands this.

Lorentz realized that the equations of electrodynamics remain independent of the velocity of the reference frame if the clocks at a distance $x$ from the origin of the frame are deemed to read the local time $t^{\prime}=t-v x / c^{2}$, where $v$ is the velocity. Lorentz assumed that this was a mathematical device that represented the inability to detect inertial motion by local experiments, with physical effects that were of first order in $v / c$. Poincaré correctly explained the meaning of Lorentz's local time as related to the synchronization of clocks done within the constraint of the principle of relativity, using the factually Galilean propagation of light (see figures 9 and 10). Poincaré remarked [30],

I suppose that observers placed in different points set their watches by means of optical signals; that they try to correct these signals by the transmission time, but that, unaware of their translational motion and thus believing that the signals travel at the same speed in both directions, they content themselves with crossing the observations, by sending one signal from A to B, then another from $\mathrm{B}$ to $\mathrm{A}$.

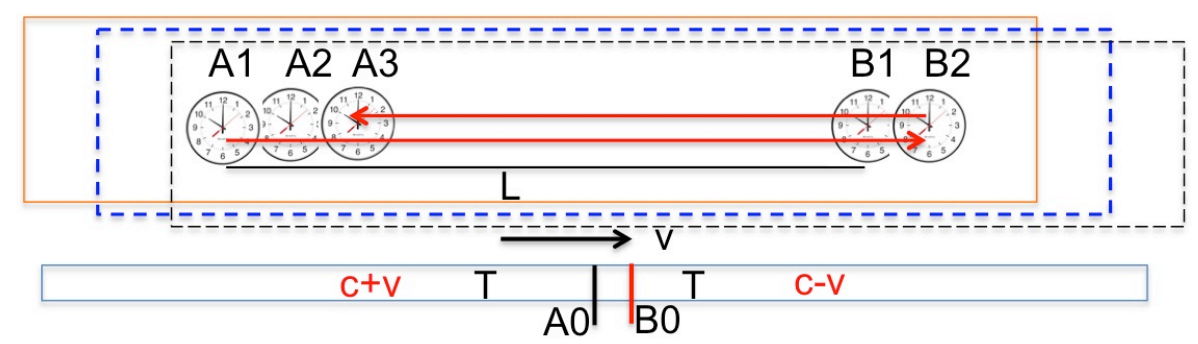

Figure 9: Synchronization by light signals in a frame that moves at velocity $v$. Observers in the frame are unaware of their motion and assume that light signals take equal times in their up and down trips, A to B and back. Clock B marks zero when it receives the light pulse and $\mathrm{A}$ marks zero at the midpoint of the total duration $2 T$. In reality, the clock moves to B2 while the signal propagates, taking more time to reach (long right arrow), and it is received back when the clock is at A3, taking less time in transit. Then the local time set at $\mathrm{B}$ is behind the time at $\mathrm{A}$, while the observers at $\mathrm{B}$ believe that they have the same time as at $\mathrm{A}$. If the separation between the clocks is $L$, the total duration $2 T=2 \mathrm{~L} / \mathrm{c}$ is divided in the ratio $t: 2 T-t=(c+v):(c-v)$. Thus $B 0$ is not the same as $A 0$; it is $\delta t=L v / c^{2}$ later than $A 0$. 


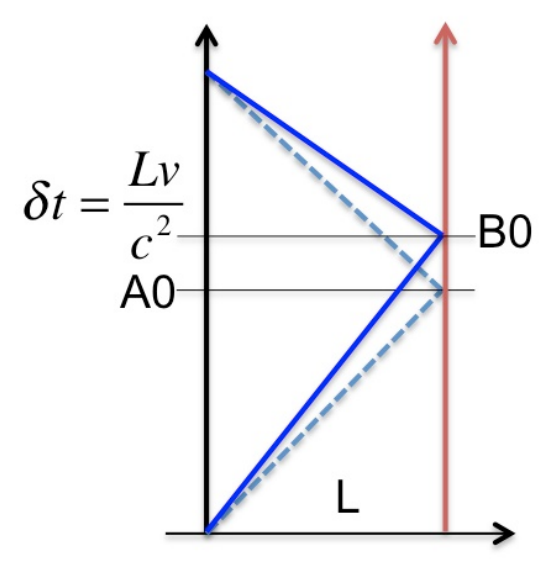

Figure 10: Clock synchronization in a space-time diagram. The dashed diagonal lines indicate the belief about the propagation of light and the blue lines indicate the Galilean reality, in a moving frame. The local time at $\mathrm{B}$ (' 0 ' of $\mathrm{B}$ ) is later by $L v / c^{2}$ relative to $\mathrm{A} 0$, which is at a distance L away.

I have italicized the essence of the interpretation. Bergson's chapter 'Half Relativity' in D-et-S, contains a clear and mathematically accurate description of this synchronization. Remarkably, Bergson's interpretation is essentially the same as Poincaré's, that the local time is the mismatch between two clocks synchronized believing that they were at rest, unaware of the motion of the frame at velocity $v$. It is obvious that the measurement of the velocity of light done using such clocks, synchronized assuming equal velocity both ways, when it is really $c-v$ and $c+v$, will always return equal (isotropic) velocity as the result.

Going further, inertially moving observers, unaware of their motion, cannot account for their own relativistic time dilation by the factor $1 / \gamma=$ $\left(1-v^{2} / c^{2}\right)^{1 / 2}$. Hence they believe that their clocks are reading the same time of those at rest, which is $\gamma$ times faster than they actually are. Thus, we get the Lorentz transformation for time, $t^{\prime 2}$ ). For space, there is no 'synchronization'; observers in motion have a different spatial coordinate, given by the Galilean formula, $x^{\prime}=x-v t$ and being insensitive to their own real length contraction by the factor $1 / \gamma$, they believe that $x^{\prime}=\gamma(x-v t)$. That is the correct physical interpretation of the Lorentz transformations.

Einstein, on the other hand, adopted the Lorentz transformations and attributed a real physical significance to the local time; this is equivalent to assuming that the velocity of light is independent of the velocity of the 
observer. Besides being inconsistent with the measurements of genuine oneway relative speeds [44], we have demonstrated the internal inconsistency in the previous appendices on time dilation.

\section{Appendix IV: Galilean Black Holes of General Relativity}

Similar to the Galilean nature of the Langevin metric in rotating frames (section 7) and the metric of an observer with proper motion velocity $v$ in FRW universe (subsection 5.2), the Painlevé representation [33] reveals the Galilean nature of the Schwarzchild black hole solution [31] of the vacuum Einstein equations $\left(R_{i k}=0\right)$ of general relativity. Though the solution was known in 1921, it was perhaps not explicitly discussed during the 1922 visit (section 6). The metric in the absence of a point mass at $r=0$ is $d s^{2} \simeq-c^{2} d t^{2}+d r^{2}+r^{2}\left(d \theta^{2}+\sin ^{2} \theta d \phi^{2}\right)$. With a mass $M$, the observer is in free fall towards the mass with radial velocity $\vec{v}=-\hat{r}(2 G M / r)^{1 / 2}$ at $r$. Galilean transformation to a uniformly accelerated frame from the homogeneous, isotropic metric is $t^{\prime}=t, r^{\prime}=r+v t$ with $v=(2 G M / r)^{1 / 2}$ and we get the metric in the observer frame from

$d s^{\prime 2}=-c^{2} d t^{2}\left(1-2 G M / c^{2} r\right)+d r^{2}+2(2 G M / r)^{1 / 2} d r d t+r^{2}\left(d \theta^{2}+\sin ^{2} \theta d \phi^{2}\right)$

Though not evident, this is the Schwarzchild metric in a non-diagonal form, without any divergence at $r=2 G M / c^{2}$. To get the metric in the usual coordinates, the $d r d t$ mixed term can be eliminated by introducing a new time coordinate defined by [54],

$$
d T=d t-d r \frac{(2 G M / r)^{1 / 2}}{c^{2}\left(1-2 G M / c^{2} r\right)}
$$

Then,

$$
\begin{aligned}
d s^{\prime 2} & =-c^{2} d T^{2}\left(1-2 G M / c^{2} r\right)+\frac{d r^{2}\left(2 G M / c^{2} r\right)}{\left(1-2 G M / c^{2} r\right)}-2(2 G M / r)^{1 / 2} d r d t \\
& +2(2 G M / r)^{1 / 2} d r d t+d r^{2}+r^{2}\left(d \theta^{2}+\sin ^{2} \theta d \phi^{2}\right) \\
& =-c^{2} d T^{2}\left(1-2 G M / c^{2} r\right)+\frac{d r^{2}}{\left(1-2 G M / c^{2} r\right)}+r^{2}\left(d \theta^{2}+\sin ^{2} \theta d \phi^{2}\right)
\end{aligned}
$$

We have the Schwarzchild line element in the standard form, obtained from a Galilean velocity transformation. 


\section{References}

[1] La théorie de la relativité, Bulletin de la Societé Francaise de Philosophie 22, 3 (1922), (Séance du 6 avril 1922).

[2] C. Nordmann, Einstein expose et discute sa théorie, Revue des Deux Mondes, Vol. IX, pp. 129-166 (1922). English translation by members of the La Rouche movement in 21st Century Science and Technology, Summer 2011.

[3] M. Biezunski, Einstein's reception in Paris in 1922, in The Comparative Reception of Relativity, T. F. Glick (Ed), 169-188 (Reidel Publishing Co. 1987).

[4] J. Canales, The Physicist and the Philosopher, (Princeton University Press, 2015).

[5] B. Latour, Some experiments in art and politics, e-flux journal no.23 (March 2011).

[6] H. Bergson, Durée et Simulanéité: à propos de la théorie d'Einstein, 1922; Duration and Simultaneity: With reference to Einstein's theory, English translation by L. Jacobson, (Bobbs-Merril Company Inc. USA, 1965).

[7] P. A. Y. Gunter, Bergson and the Evolution of Physics, University of Tennessee Press, Knoxville, 1969.

[8] H. A. Lorentz, Electromagnetic phenomena in a system moving with any velocity smaller than that of light, Proceedings of the Royal Netherlands Academy of Arts and Sciences 6, 809-831 (1904).

[9] H. Poincaré, La théorie de Lorentz et le principe de réaction, Archives Néerlandaises des Sciences Exactes et Naturelles, 2ème série 5, 252-278 (1900).

[10] A. Einstein, On the electrodynamics of moving bodies (in German), Annalen der Physik 17, 891-921 (1905).

[11] A. A. Michelson and E. W. Morley, On the relative motion of the earth and the luminiferous ether, American Journal of Science 34, 333-345 (1887). 
[12] H. Poincaré, Sur la dynamique de l'électron, Comptes Rendus de l'Acad. des Sc. 14 1504-1508 (1905).

[13] M. Planck, Principle of Relativity, in Eight Lectures on Theoretical Physics, Columbia University 1909 (Translated by A. P. Wills, Columbia University Press, 1915).

[14] A. A. Michelson, Studies in Optics (The University of Chicago Press, 1927).

[15] M. Ruderfer, First order terrestrial ether drift using the Mössbauer radiation Phys. Rev. Letters 5, 191 (1960).

[16] M. Ruderfer, Erratum: First order terrestrial ether drift using the Mössbauer radiation, Phys. Rev. Letters 7, 361 (1961).

[17] Champeney D C, Isaak G R and Khan A M 1963 An Aether Drift Experiment Based on the Mössbauer Effect, Phys. Lett. 7 241-243.

[18] Krisher T P et al. 1980 Test of the Isotropy of the One-way Speed of Light Using Hydrogen-Maser Frequency Standards Phys. Rev. 42 731734 .

[19] A. Einstein, Relativity: The Special and General Theory (1916), translation by R. W. Lawson, London: Methuen \& Co. Ltd, London, 1920.

[20] Relativity beneath the layers of logic, to be published (2019).

[21] H. Minkowski, Das Relativitätsprinzip, Annalen der Physik. 352, 927 938 (1907, published in the journal in 1915)

[22] P. Langevin, L'évolution de l'espace et du temps, Scientia 5, 31 (1911).

[23] A. Einstein, Dialogue about objections to the theory of relativity, Die Naturwissenschaften 6, 697 (1918). The English translation of the original German version is available in the translated companion volume of The Collected Papers of Albert Einstein, Volume 7, The Berlin Years: Writings, 1918-1921, pp. 66-75 (Princeton University Press, Princeton, NJ, 2002). 
[24] H. Bergson, The journey in the projectile, first appendix to Duration and Simultaneity, second edition (1923). Contents of J. Becquerel's letter is reproduced without revealing the name.

[25] H. J. Bhabha, Nuclear forces, heavy electrons, and the $\beta$-decay, Nature 141, 117 (1938); this paper was written when muons were misidentified with the Yukawa particles and it was the first time such a decaying nuclear particle was treated as a relativistic clock.

[26] B. Rossi and D. B. Hall, Variation of the rate of decay of Mesotrons with momentum, Phys. Rev. 59, 223 (1941).

[27] C. S. Unnikrishnan, On Einstein's resolution of the twin clock paradox, Current Science 89, 2009 (2005).

[28] J. C. Hafele and R. E. Keating, Around-the-World atomic clocks: Predicted relativistic time gains, Science 177, 166-168 (1972); Around-theworld atomic clocks: Observed relativistic time gains, Science 177, 168170 (1972).

[29] L. Essen, The Special Theory of Relativity: a Critical Analysis, Clarendon Press (1971).

[30] H. Poincaré, The Principles of Mathematical Physics, Lecture at the International Congress of Arts and Science, St. Louis, September, 1904, published in The Monist, XV, 1 (1905).

[31] K. Schwarzchild, Über das gravitationsfeld eines massenpunktes nach der Einsteinschen theorie, Sitzungsberichte der Königlich Preussischen Akademie der Wissenschaften 7, 189-196 (1916).

[32] S. Chandrasekhar, The maximum mass of ideal white dwarfs, Astrophysical Journal 74, 81-82 (1931).

[33] P. Painlevé, La mécanique classique et la théorie de la relativité, C. R. Acad. Sci. (Paris) 173, 677-680 (1921).

[34] A. Kastler, Albert Einstein, à propos du centenaire de sa naissance, Mémoires de la classe de sciences, Académie Royal de Belgique, 2 séries, 44, 13-27 (1981). 
[35] G. Sagnac, L'éther lumineux démontré par l'effet du vent relatif d'éther dans un interféromètre en rotation uniforme, C. R. Acad. Sci. Paris 157, 708-710 (1913).

[36] G. Sagnac, Sur la preuve de la réalité de l'éther lumineux par l'expérience de l'interférographe tournant, C. R. Acad. Sci. Paris 157 1410-1413 (1913).

[37] O. Darrigol, Georges Sagnac: A life for optics, C. R. Physique 15 789840 (2014).

[38] A. A. Michelson. Relative motion of earth and aether. Philosophical Magazine. 8, 716-719 (1904).

[39] A. A. Michelson, The effect of the earth's rotation on the velocity of light, I. Astrophysical Journal 61137 (1925); A. A. Michelson and H. G. Gale, The effect of the earth's rotation on the velocity of light, II". Astrophysical Journal 61, 140 (1925).

[40] O. Lodge, VI. Experiments on the absence of mechanical connexion between ether and matter, Phil. Trans. Royal Soc. A 189, 149-166 (1897).

[41] Letter dated 26 June 1922 from Paul Epstein to A. Einstein, Document No. 251 in The Collected Papers of A. Einstein, Volume 13: The Berlin Years: Writings \& Correspondence January 1922-March 1923 (Princeton University Press, 2012)

[42] L. Silberstein, The propagation of light in rotating systems, Jl. Opt. Soc. America 5, 291 (1921).

[43] P. Langevin, Sur la théorie de la relativité et l'expérience de M. Sagnac, C. R. Acad. Sci.. 173, 831-834 (1921); Sur l'expérience de M. Sagnac, C. R. Acad. Sci. 205, 304-306 (1937).

[44] C. S. Unnikrishnan, Physics in the 'Once-Given' Universe, in Recent Advances in Theoretical Physics (S. Kar and G. Ghosh (eds), World Scientific, 2009), pp 99-120.

[45] L. H. Thomas, Motion of the spinning electron, Nature. 117, 514 (1926). 
[46] E. Mach, The Science of Mechanics, Chapter 2, section VI (Newton's views of time, space and motion), English translation: T. J. McCormack (Open Court Publishing Co. Chicago, 1893).

[47] D. W. Sciama, On the origin of inertia, MNRAS 113, 34-42 (1953).

[48] E. Hubble, Extragalactic nebulae, Astrophysical Journal 64, 321-369 (1926); A relation between distance and radial velocity among extragalactic nebulae, PNAS 15, 168-173 (1929).

[49] C. S. Unnikrishnan, Cosmic Relativity: the theory, its implications and experimental tests, gr-qc/0406043 (a preliminary version, which has evolved considerably).

[50] C. S. Unnikrishnan, Experimental evidence for the gravitational basis for relativity and dynamics, in S. M. Wagh, S. D. Maharaj and G. Chon (Eds), The Physical Universe (Central India Research Institute, 2018) pp 183-202.

[51] C. S. Unnikrishnan, Precision measurement of the one-way speed of light and implications to the theory of relativity, in Physical Interpretations of Relativity Theory: Proceedings, London 2006 (M. C. Duffy, V. O. Gladyshev, A. Morozov, and P. Rowlands (Eds.), Bauman Moscow State Technical University 2011), pp. 505-511.

[52] A. Friedman, Z. Phys. 10, 377-386 (1922): On the Curvature of Space (English translation, Gen. Relativ. Gravit. 31, 1991-2000 (1999).

[53] C. S. Unnikrishnan, Compatibility of duration and simultaneity of the Bergsonian philosophy with the time of physics, to be published (2020).

[54] E. F. Taylor and J. A. Wheeler, Exploring Black Holes: Introduction to General Relativity, (Addison Wesley Longman Inc. 2000). 